\title{
Multiplexed imaging of human tuberculosis granulomas uncovers immunoregulatory features conserved across tissue and blood
}

Erin F. McCaffrey ${ }^{1}$, Michele Donato ${ }^{2,3}$, Leeat Keren ${ }^{1}$, Zhenghao Chen ${ }^{4}$, Megan Fitzpatrick ${ }^{5}$, Vladimir Jojic ${ }^{4}$, Alea Delmastro ${ }^{1}$, Noah F. Greenwald ${ }^{1}$, Alex Baranski ${ }^{1}$, William Graf, Marc Bosse ${ }^{1}$, Pratista K. Ramdial7,8, Erna Forgo ${ }^{1}$, David Van Valen ${ }^{6}$, Matt van de Rijn ${ }^{1}$, Sean C. Bendall ${ }^{1}$, Niaz Banaei ${ }^{1,9}$, Adrie J.C. Steyn ${ }^{6,10}$, Purvesh Khatri2,3, \& Michael Angelo ${ }^{1}$

'Stanford University Department of Pathology; ${ }^{2}$ Stanford Center for Biomedical Informatics Research; ${ }^{3}$ Stanford Institute for Immunity, Transplantation and Infection; ${ }^{4}$ Calico Life Sciences LLC; ${ }^{5}$ University of Wisconsin, Department of Pathology; ${ }^{6}$ Division of Biology and Bioengineering, California Institute of Technology; ${ }^{7}$ African Health Research Institute; ${ }^{8}$ University of KwaZulu-Natal, Inkosi Albert Luthuli Central Hospital ${ }^{9}$ Stanford University Department of Medicine-Infectious Diseases; ${ }^{10}$ Department of Microbiology, University of Alabama at Birmingham

\section{Abstract}

Tuberculosis (TB) is an infectious disease caused by Mycobacterium tuberculosis that is distinctly characterized by granuloma formation within infected tissues. Granulomas are dynamic and organized immune cell aggregates that limit dissemination, but can also hinder bacterial clearance. Consequently, outcome in TB is influenced by how granuloma structure and composition shift the balance between these two functions. To date, our understanding of what factors drive granuloma function in humans is limited. With this in mind, we used Multiplexed lon Beam Imaging by Time-of-Flight (MIBI-TOF) to profile 37 proteins in tissues from thirteen patients with active TB disease from the U.S. and South Africa. With this dataset, we constructed a comprehensive tissue atlas where the lineage, functional state, and spatial distribution of 19 unique cell subsets were mapped onto eight phenotypically-distinct granuloma microenvironments. This work

revealed an immunosuppressed microenvironment specific to TB granulomas with

32 spatially coordinated co-expression of IDO1 and PD-L1 by myeloid cells and proliferating

33 regulatory $T$ cells. Interestingly, this microenvironment lacked markers consistent with T-

34 cell activation, supporting a myeloid-mediated mechanism of immune suppression. We

35 observed similar trends in gene expression of immunoregulatory proteins in a 36 confirmatory transcriptomic analysis of peripheral blood collected from over 1500 
37 individuals with latent or active TB infection and healthy controls across 29 cohorts spanning 14 countries. Notably, PD-L1 gene expression was found to correlate with TB progression and treatment response, supporting its potential use as a blood-based biomarker. Taken together, this study serves as a framework for leveraging independent cohorts and complementary methodologies to understand how local and systemic

42 immune responses are linked in human health and disease.

\section{Introduction}

Mycobacterium tuberculosis (Mtb) is the leading cause of mortality from infectious disease in the world, accounting for nearly 1.5 million deaths each year ${ }^{1}$. Relative to other communicable diseases, the reduction in the incidence of TB infection over the last 20 years has been modest. This is largely due to the lack of a highly efficacious vaccine, lengthy and toxic antimicrobial regimens, and emergence of multidrug resistance. Along these lines, previous efforts to develop new host-directed therapies have been hindered by an incomplete understanding of how TB interacts with the human immune system

52 during infection.

Infection is initiated when bacteria are engulfed by alveolar macrophages or other resident phagocytes after being inhaled into the lungs ${ }^{2,3}$. This triggers an immune response that ultimately converges on formation of a granuloma, a dynamic and spatiallyorganized tissue structure comprised of macrophages, granulocytes, lymphocytes, and

57 fibroblasts. A prototypical non-necrotic granuloma consists of a myeloid-predominant central core region that is enriched with infected macrophages and encircled by lymphocytes. From the perspective of facilitating an effective host response, granulomas play seemingly contradictory roles. On the one hand, consolidation of infected cells within

61 the myeloid core limits dissemination by partitioning them away from uninvolved lung

62 parenchyma. On the other, tolerogenic pathways that are upregulated within this region 63 limit bacterial clearance ${ }^{4-6}$.

64 Depending on the histological subtype and bacterial burden, granuloma 65 composition can be highly variable? ${ }^{7}$. This variability can manifest within a single individual, 
67 immunological features that each progress independently of one another over time ${ }^{8}$. 68 Controlled infections in non-human primates (NHP) have revealed that a single individual 69 can possess well over ten granulomas and that the inflammatory profile, size, and bacterial ecology of these lesions can vary dramatically ${ }^{9-11}$. Thus, the trajectory of each

71 granuloma can vary across a spectrum between complete bacterial clearance to 72 uncontrolled dissemination. This discordance suggests that local host-bacterial dynamics

73 within the tissue microenvironment play a central role in determining granuloma fate. 74 Along these lines, a growing number of studies suggest that granuloma structure and 75 immune cell function are interconnected ${ }^{12,13}$. For example, previous work has suggested 76 that impaired adaptive immunity might be the consequence of $\mathrm{T}$ cells being largely excluded from the myeloid-dominated central core region where infected macrophages 78 tend to localize ${ }^{14,15}$.

Taken together, these findings suggest that TB disease progression is heavily impacted by focal, spatially-encoded regulatory mechanisms within the granuloma

81 microenvironment. Consequently, understanding how these mechanisms promote

82 bacterial clearance or persistence is critical for designing effective therapies that promote 83 long term immunity. With this in mind, we used Multiplexed Ion Beam Imaging by Time84 of-Flight (MIBI-TOF) to simultaneously image 37 proteins at subcellular resolution in solid 85 tissue from individuals with active TB infection ${ }^{16}$. We compared granuloma composition with respect to 19 unique cell subsets to delineate different subtypes of granulomas that were enriched for classical monocytes, myeloid-derived suppressor cell-like (MDSC) macrophages, or tertiary lymphoid structures (TLS). We then utilized an adaptation of Latent Dirichlet Allocation (spatial-LDA) to identify spatially-coordinated immune responses within eight recurrent cellular microenvironments. These analyses revealed a

91 microenvironment characterized by expression of regulatory proteins, IDO1 and PD-L1, 92 and proliferative regulatory T cells. Paradoxically, these cells were not accompanied by 93 significant numbers of PD-1+ lymphocytes or any other evidence suggesting $\mathrm{T}$ cell 94 exhaustion.

To determine if these features were associated with drug treatment or severity of infection, we leveraged publicly available gene expression data of peripheral blood from 
97 patients with TB. In line with the granuloma imaging data, we found increased expression

98 of IDO1 and CD274 (PD-L1) and diminished expression of PDCD1 (PD-1) and LAG3 in

99 individuals with active TB. Moreover, CD274 (PD-L1) gene expression was found to

100 associate with progression from latent to active disease and with therapeutic response,

101 suggesting it could be used as a novel prognostic biomarker. Taken together, this work

102 provides compelling evidence for a myeloid-mediated mechanism of immune suppression

103 driven locally within the granuloma that promotes bacterial persistence and subverts T-

104 cell activation.

105

\section{Results}

Multiplexed imaging of human tuberculosis granulomas reveals structured immune cell composition

To assess granuloma composition and architecture in TB, we curated a cohort of actively infected human tissues. Archival formalin-fixed paraffin-embedded (FFPE) specimens from patients treated in the United States or South Africa were procured from

112 Stanford Health Care or University of KwaZulu-Natal, Inkosi Albert Luthuli Central 113 Hospital, respectively (Extended Data Table 1). The South African samples were 114 pulmonary tissues from patients that underwent therapeutic resection for advanced TB (n $115=6$ ). While TB disease typically manifests in the lung, infection can disseminate to extra116 pulmonary sites as well ${ }^{17,18}$. To characterize TB infection at an earlier stage and assess 117 how granuloma composition varies with infection site, samples from US patients 118 consisted of diagnostic biopsies from lung $(n=2)$, pleural cavity $(n=3)$, lymph node $(n=$ 119 1), and endometrium $(n=1)$ (Fig. 1a).

120 Tissue sections for each specimen were reviewed by an anatomic pathologist and 121 screened to include the presence of solid, non-necrotic granulomas or active 122 granulomatous inflammation, while excluding excessively necrotic or fibrotic regions 123 (Extended Data Fig. 1a). MIBI-TOF was subsequently used to image two $500 \mu \mathrm{m}^{2}$ fields 124 of view (FOVs) per tissue stained with a 37-plex panel of metal-labeled antibodies (Fig. 125 1b, Extended Data Fig. 1b-c, Extended Data Table 2) ${ }^{16}$. The antibody panel included 126 markers to comprehensively phenotype most major immune and non-immune cell 
127 lineages, including lymphocytes, macrophages, granulocytes, stroma, and epithelium.

128 The panel also included antibodies for 12 functional markers with an emphasis on those 129 with well-documented immunoregulatory pathways, including PD-1, Lag3, PD-L1, and 130 IDO1.

131 To extract single cell phenotypes, multiplexed imaging data were processed with 132 a low-level pipeline prior to single-cell segmentation (Fig. 1a, Extended Data Fig. 1d) ${ }^{19-}$ 13321 . Each FOV contained an average of $\sim 1400$ single cells ( $s d=312$ ) (Extended Data Fig. 134 2d). Hierarchical application of the FlowSOM algorithm (Extended Data Fig. 2a, b) was 135 employed to phenotype 19 unique cell subsets (Fig. 1c) ${ }^{22}$ using cell area normalized 136 values of protein expression for each segmented cell event. For each image, FlowSOM 137 clusters and segmentation masks were combined to generate cell phenotype maps 138 (CPM) where each cell is labeled by its phenotype (Fig. 1d, Extended Data Fig. 2c).

Consistent with previous work, granuloma composition was predominated in most 140 lesions by $T$ cells and myeloid cells, (average myeloid: lymphoid ratio $=1.4$, sd $=1.0)^{23}$. 141 Myelomonocytic cells were comprised of multiple subsets of macrophages, dendritic cells, 142 and monocytes that were each distinguished by varying degrees of co-expression of 143 CD11c, CD11b, CD209, CD68, CD14, CD16, and CD206 (Fig. 1e). Granulocytes were 144 comprised largely of neutrophils (mean $=1.2 \%, \mathrm{sd}=1.7)$ and mast cells $(0.6 \% \pm 0.8)^{24-}$ 14526 . We also identified $\gamma \delta$ T cells $(0.1 \% \pm 0.22)$, CD209+ dendritic cells $(0.2 \% \pm 0.5)$, and 146 regulatory $\mathrm{T}$ cells $(0.4 \% \pm 0.6)$, highlighting the capability of our approach to enumerate 147 low abundance immune cell populations that have been suggested to play a key role in 148 granuloma pathology. In line with reports of increased vascularization in active disease, 149 non-immune cells were predominated by endothelial cells $(5.7 \% \pm 3.1)$ while fibroblasts $150(3.3 \% \pm 5.1)$ and epithelial cells $(2.7 \% \pm 4.0)$ varied significantly between lesions 151 (Extended Data Fig. 2e, f) 27,28. Altogether, we assigned 94\% ( $n=33,194$ single cells) of 152 cells to 19 subsets that in aggregate ranged in frequency from $0.1-15 \%$ across our 153 dataset. 

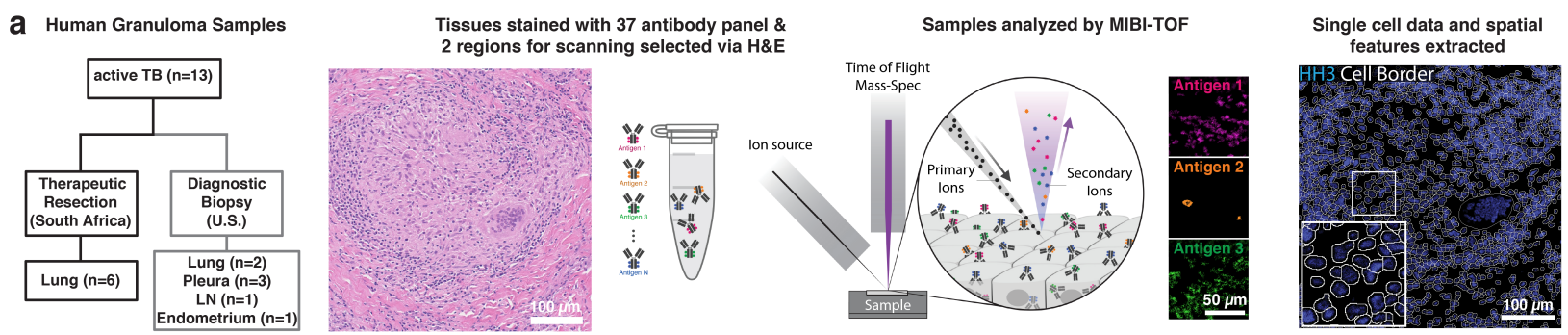

b Patient 8 ROI 1- Pulmonary TB
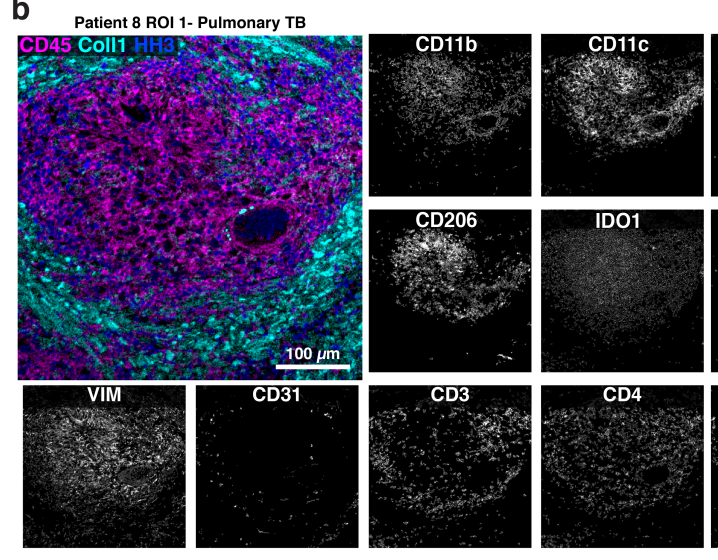

d

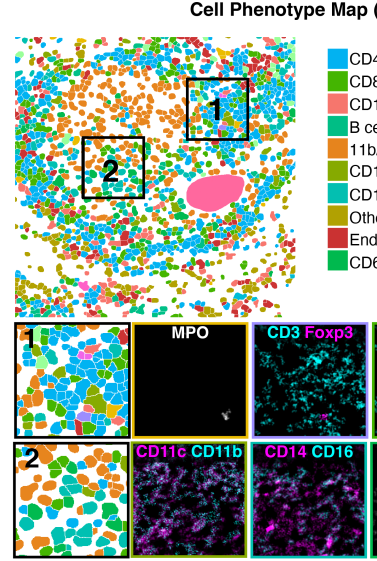

f Macrophage \& monocyte frequency across clusters
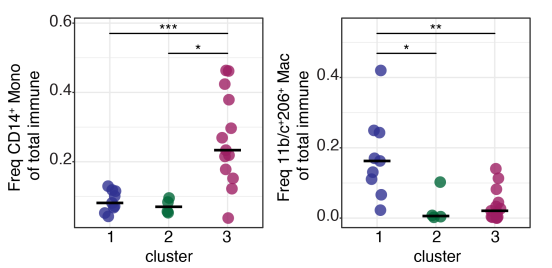

$2^{2}$

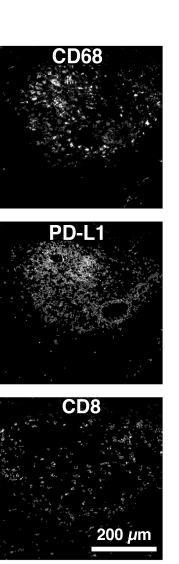

C
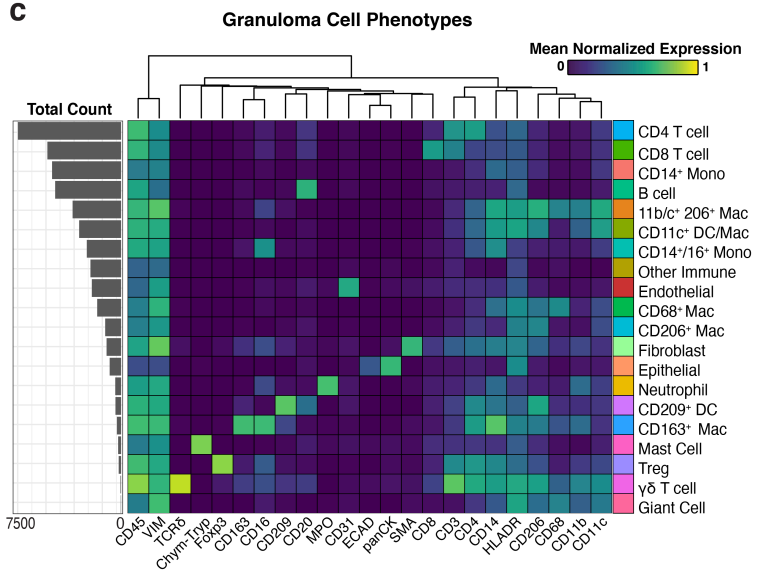

e

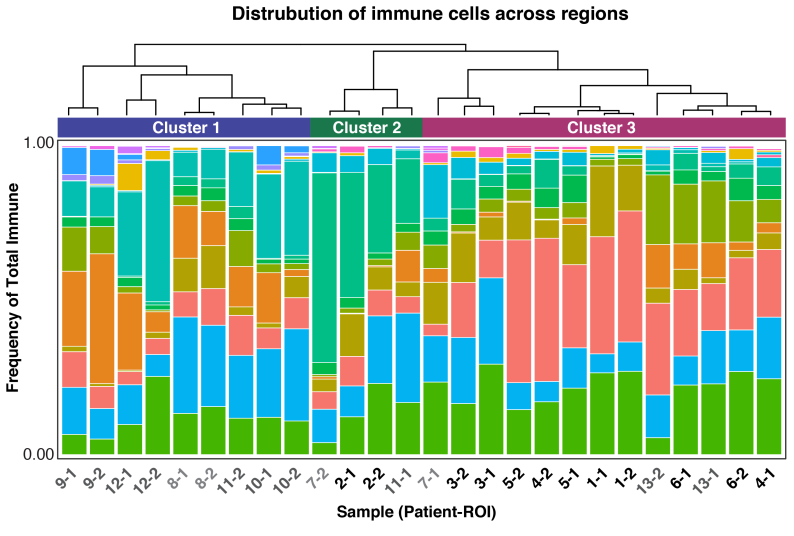

$\mathbf{h}$

CD4 T cell \& CD8 T cell frequency across clusters

g CD4 $\mathrm{T}$ cell : CD8 $\mathrm{T}$ cell ratio across sample

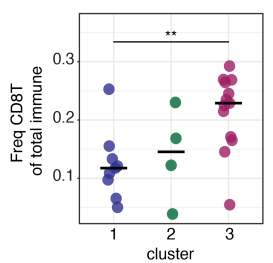

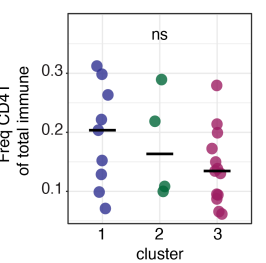

Figure 1. Multiplexed imaging of human tuberculosis granulomas reveals structured immune cell composition. (a) Conceptual overview of MIBI-TOF analysis of human TB granulomas. (b) Representative images from a pulmonary TB section. (c) Cell lineage assignments based on normalized expression of lineage markers (heatmap columns). Rows are ordered by absolute abundance shown on the bar plot (left), while columns are hierarchically clustered (Euclidean distance, average linkage). (d) Cell identity overlaid onto the segmentation mask for a representative pulmonary TB section (left). Two insets (bottom) are shown. (e) The relative abundance of immune cell types across all TB FOVs with 
cell types ordered by decreasing median abundance and bars ordered by the hierarchical relationship of pairwise Pearson correlation coefficients (distance $=1-$ correlation, complete linkage). Consensus clusters are annotated above bar plot (cluster 1 = blue, cluster 2 = green, cluster $3=$ purple). (f) Frequency of CD14+ monocytes and $11 \mathrm{~b} / \mathrm{c}^{+} 206^{+}$ macrophages of total immune cells colored by cluster. Line represents the median. $(\mathbf{g})$ The CD4 T: CD8 T cell ratio represented as a log2 fold-change for each TB FOV (top) colored by cluster. (h) Frequency of CD4+ and CD8 ${ }^{+} \mathrm{T}$ cells of total immune cells colored by cluster. Line represents the median. All p-values determined with a Wilcoxon Rank Sum Test where: ns $p>0.05,{ }^{\star} p<0.05,{ }^{\star \star} p<0.01,{ }^{\star \star \star} p<0.001$.

To assess whether granulomas can be grouped into subtypes based exclusively on immune cell subset frequencies, we clustered FOVs using Pearson correlation based on their immune cell proportions (Extended Data Fig. $2 \mathrm{~g}$ ). We found that using three clusters explained $72 \%$ of the variance in our dataset (Fig. 1e, Extended Data Fig. 2h). Cluster 1 (Fig. 1e, f) was characterized by CD11 $\mathrm{C}^{+} \mathrm{CD} 11 \mathrm{~b}^{+}$macrophages, intermediate monocytes, and occasional CD163+ macrophages. Chi-square analysis of cell type cooccurrence showed significant associations of $\mathrm{CD}_{163^{+}}$macrophages with intermediate monocytes (adj. $p=0.0090$ ), regulatory T cells (Tregs) (adj. $p=0.003$ ), and fibroblasts (adj. $p=0.02$ ), suggesting coordinated cellular presence in this cluster (Extended Data Fig. 2i). Cluster 2 was enriched for B cells (Fig. 1e). Lastly, a subset of granulomas (cluster 3) was enriched for classical monocytes (Fig. 1e, f) and higher numbers of CD8+ T cells, resulting in a skewed $\mathrm{CD}^{+}$to $\mathrm{CD}^{+} \mathrm{T}$ cell ratio relative to clusters 1 and 2 . Since most samples in cluster 3 were surgical lobectomies from patients in South Africa, this profile could be related to disease severity, comorbidity, or mandatory pre-surgical antimicrobial therapy. Given this result, we analyzed therapeutic resections with respect to HIV status (Extended Data Fig. 3). While we observed a slight difference in the CD4+

171 to $\mathrm{CD}^{+} \mathrm{T}$ cell ratio (approximately two-fold decrease in HIV+ specimens, $\mathrm{p}=0.026$ ), we 172 found no material differences in immune cell frequencies that define these clusters. Taken

173 together, this comprehensive cell census reveals distinct types of granulomas that are defined by immune cell frequency and associate with TB disease status.

\section{Spatial analysis of granulomas identifies organized protein expression patterns and} conserved cellular microenvironments

In order to identify recurrent patterns of protein co-expression in TB granulomas, we conducted a spatial enrichment analysis that quantified the degree of co-occurrence between pairs of proteins relative to a null distribution (Extended Data Fig. 4a) ${ }^{19}$. Pairwise 
181 enrichment scores for each protein pair were then used to construct an interaction 182 network that was subsequently analyzed using a community detection algorithm ${ }^{29}$ (Fig. $1832 \mathrm{2a}$ ). This analysis resulted in three spatial modules consistent with canonical granuloma 184 structures, including the myeloid core, lymphocytic cuff, and stromal compartment. 185 Intriguingly, these modules also revealed more granular, previously unknown features 186 linking cell function to spatial organization, such as the association of the lymphocytic cuff with H3K9Ac and the myeloid core with IDO1 and PD-L1 (Fig. 2a).

These findings motivated us to examine how the local cellular neighborhood relates to single cell function and granuloma structure. Therefore, we employed spatial 190 Latent Dirichlet Allocation (spatial-LDA) ${ }^{30}$ to discover and assign cellular 191 microenvironments (MEs) to each cell in a CPM, where an ME is defined by a set of cell 192 types found to be spatially co-occurring across the cohort. Using this approach, we 193 identified eight MEs for summarizing the local frequency of cell subsets within a $50 \mu \mathrm{m}$ 194 radius of a target cell (Fig. $2 b)$. We then labeled each cell with its highest probability ME to generate a maximum probability ME map (MaxPM, Fig. 2c, Extended Data Fig. 4b).

196 Through this approach, granuloma composition and structure can be summarized with 197 two complementary and simplified spatial representations: a CPM and MaxPM where 198 cells are labeled either by cell type or by ME, respectively (Fig. 2c).

This allowed us to annotate well known features of granuloma histology in an 200 unbiased fashion, while also revealing previously unrecognized cellular niches (Fig. 2d, 201 e). For example, the large majority of granuloma macrophages and monocytes were

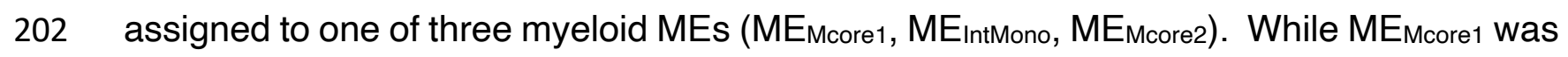
203 found to some degree across all specimen types, $\mathrm{ME}_{\text {IntMono }}$ and $\mathrm{ME}_{\mathrm{Mcore}}$ were significantly 204 enriched in either extra-pulmonary diagnostic biopsies or therapeutic resections, 205 respectively (Fig. 2f, Extended Data Fig. 4d, e). MEMcore1 exhibited the strongest 206 preference for the histologically defined granuloma core region (median frequency in core

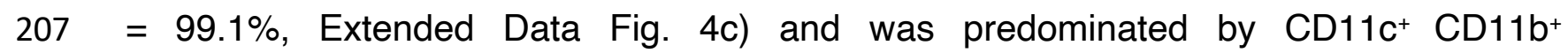
208 macrophages (Fig. 2d, e). MEMcore2 exhibited myeloid core preference to a lesser extent 209 and was enriched for CD14+ classical monocytes (median frequency in core $=60.9 \%$, 
210 Extended Data Fig. 4c). Lastly, ME IntMono exhibited low myeloid core preference and was 211 enriched for CD14+ CD16+ intermediate monocytes (Fig. 2d, e).

$212 \quad E_{L c u f f}$ aligned with the second histologically defined microenvironment in the 213 granuloma, the lymphocytic cuff, and was comprised predominantly of CD4+ and CD8+ T 214 cells (Fig. 2d, e). METLS is a second lymphoid ME that is B cell predominated with sparse 215 numbers of follicular helper $\mathrm{T}$ cells $\left(\mathrm{CD} 4^{+} \mathrm{PD}-1^{+}\right)$, consistent with tertiary lymphoid 216 structures (TLS, confirmed by H\&E in serial sections, Fig. 2e) ${ }^{31-33}$. This ME was highly 217 abundant in FOVs that were B cell predominated from cell frequency cluster 2 (Fig. 2f, 218 Fig. 1e).

As previously demonstrated, some granulomas exhibited a fibrotic wound healing response consisting of fibroblasts and $\mathrm{CD}_{163}{ }^{+} \mathrm{M} 2$-like macrophages (Fig. 1e). These 221 cells were found to co-localize within $\mathrm{ME}_{\mathrm{Fib}}$, where CD36, a fibroblast marker, and 222 collagen-1, a marker for fibrosis are expressed (Fig. 2d, e) ${ }^{34}$. The last two MEs 223 represented less characterized cellular environments in TB infection. MEvasc was

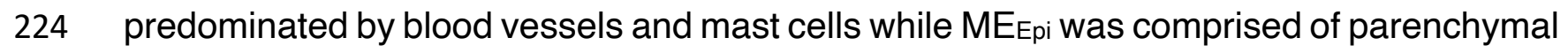
225 epithelial cells and CD206+ alveolar-like macrophages (Fig. 2d, e). Given that they are known to participate in angiogenesis, tissue repair, and immune cell recruitment ${ }^{35}$, 227 perivascular localization of mast cells in the granuloma could suggest their involvement 228 in some of these processes. On the other hand, since MEvasc was found to be lower in 229 extra-pulmonary biopsies (Fig. 2f, Extended Data Fig. 4e), this may reflect organ-specific 230 differences in vascularity and abundance of tissue resident mast cells.

We next sought to compare sample composition with respect to ME frequency.

232 Using a correlation-based approach, we found that five ME frequency clusters accounted 233 for $89 \%$ of variance (Fig. 2f, Extended Data Fig. 4f). Notably, two of these clusters were 234 comprised of samples from more than one cell frequency cluster (as defined in Fig. 1e). 235 These clusters, along with a Principle Component Analysis (PCA) of all samples based 236 on mean ME probability, further supported tissue site and clinical cohort-associated ME 237 profiles (Fig. 2f, Extended Data Fig. 4g). Altogether, this suggests that MEs capture 238 recurrent spatial features of granulomas that are not discernible by bulk cell composition 239 alone. 
a Community structure of protein spatial enrichment network

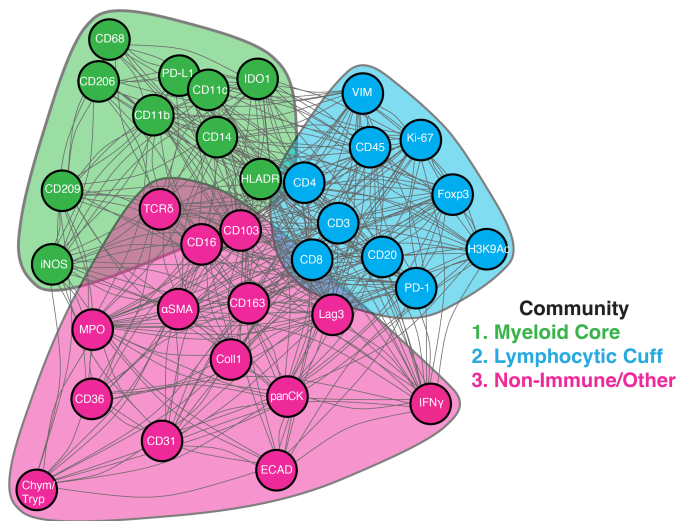

b
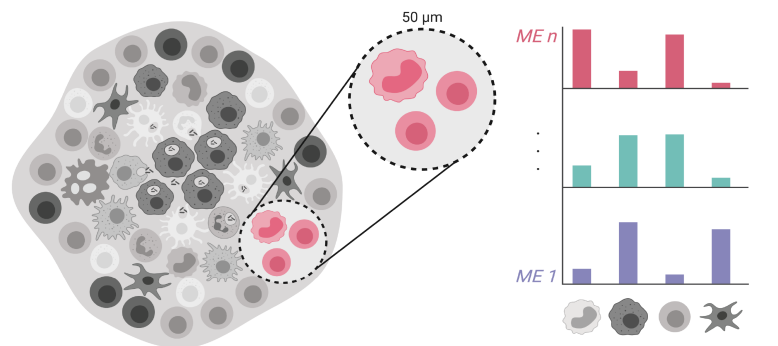

Microenvironment (ME) $=$ cell subsets cooccuring in a spatially defined proximity
C

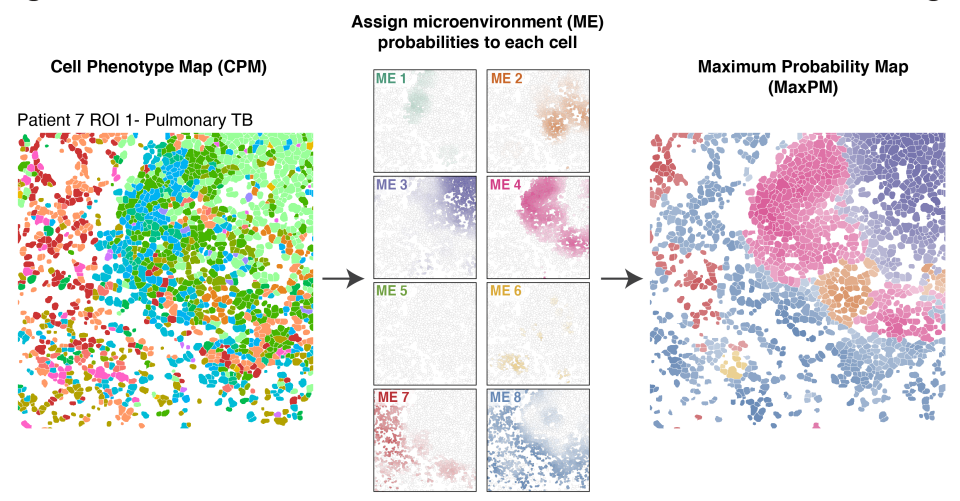

d

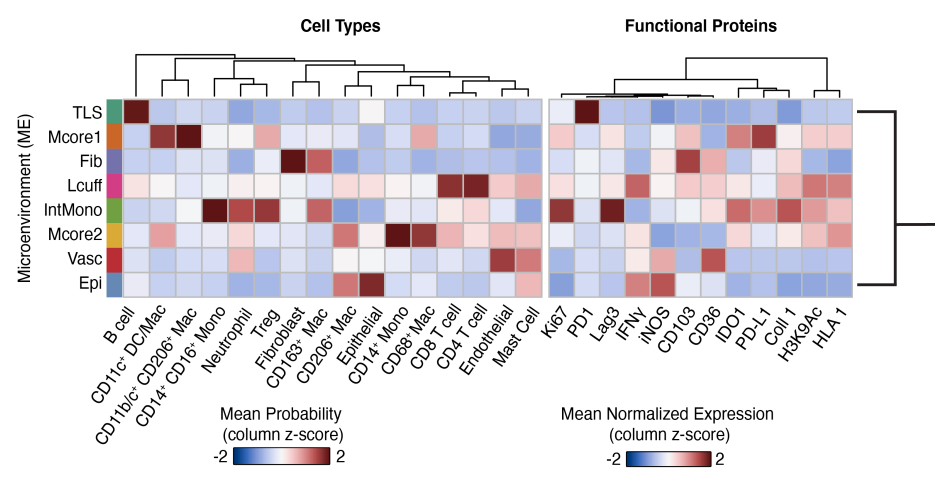

f

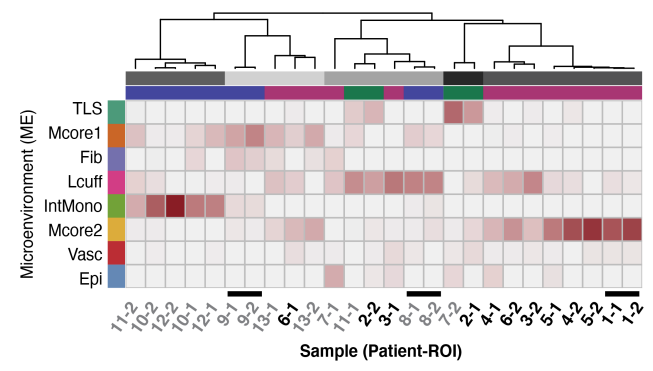

Frequency of Total

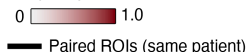

Therapeutic Resection Diagnostic Biopsy

ME Cluster 1

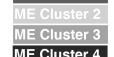
\begin{tabular}{l} 
Cell Cluster 1 \\
Cell Cluster 2 \\
\hline Cell Cluster
\end{tabular} \begin{tabular}{|l|l|l} 
ME Cluster 4 \\
\hline ME Cluster 5
\end{tabular} e $M E_{\text {Mcore }} M_{\text {IntMono }} M E_{M c o r e 2}-$ Myeloid core and granuloma monocytes
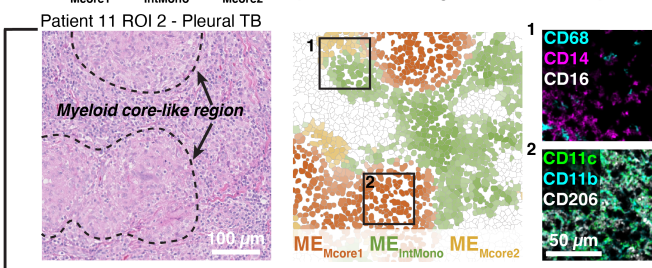

$\mathrm{ME}_{\text {Lcuft }}$ - Lymphocytic Cuff

Patient 8 ROI 1 - Pulmonary TB
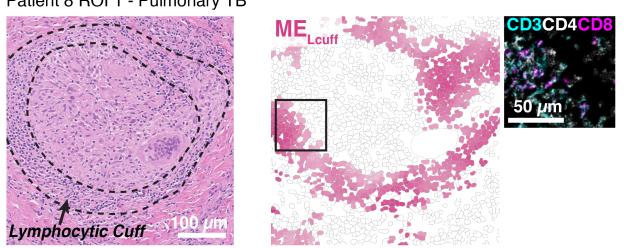

$\mathrm{ME}_{\mathrm{TLS}}$ - Tertiary lymphoid structure (TLS)

Patient 7 ROI 2 - Pulmonary TB
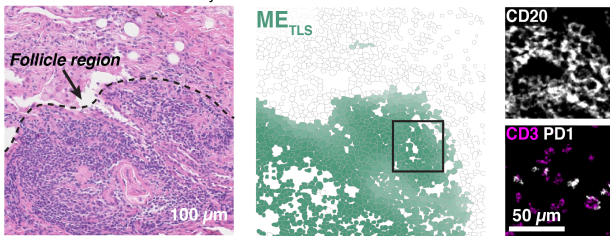

$\mathrm{ME}_{\mathrm{Fib}}-$ Fibrosis

Patient 9 ROI 1-Pleural TB
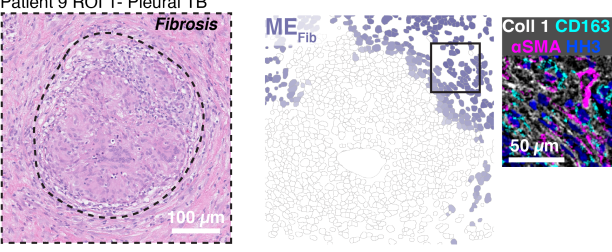

$\mathrm{ME}_{\text {vasc }}$ - Vascular Mast Cells $\mathrm{ME}_{\mathrm{Epi}}$ - Lung parenchyma

Patient 2 ROI 1- Pulmonary TB Patient 3 ROI 1- Pulmonary TB
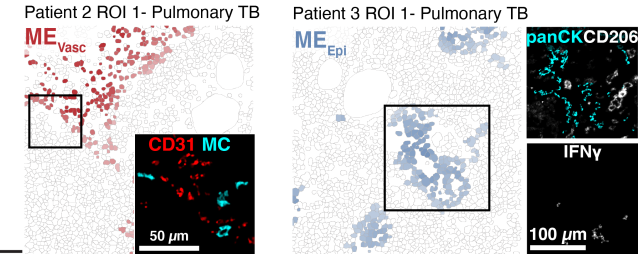

Figure 2. Spatial analysis of granulomas identifies organized protein expression patterns and conserved cellular microenvironments. (a) Positive spatial enrichments (average z-score $>0$ ) between protein pairs as a 
weighted, undirected network (edge weight is proportional to average z-score) with three communities (myeloid core = green, lymphocytic cuff $=$ blue, non-immune/other $=$ pink). (b) Conceptual overview of spatial-LDA. (c) Cell probability map (left), max probability map (right), and microenvironment (ME) probability for 8 MEs (middle, scaled $0-1$ ) for a pulmonary TB section. (d) Heatmap of ME preferences for all subsets (standardized mean ME loading) with hierarchical clustering (Euclidean distance, complete linkage) and mean normalized expression of functional markers (probability weighted mean) with columns hierarchically clustered (Euclidean distance, complete linkage). (e) Biological classification of microenvironments. (f) Frequency of all MEs per FOV. Heatmap columns are hierarchically clustered (Pearson correlation, complete linkage). Paired ROls from the same patient annotated with a black bar. ME and cell composition clusters annotated below dendrogram. Cell cluster annotation is based on clusters in Fig. 1e.

Granuloma myeloid cells are characterized by spatially coordinated expression of IDO1 and PD-L1

Our microenvironment and spatial protein network modeling revealed that myeloidrich regions in the granuloma are characterized by expression of two proteins, IDO1 and PD-L1 (Fig. 2a, d). Given the tolerogenic role of these proteins ${ }^{36-41}$, we sought to identify myeloid cell subsets that could promote bacterial persistence through upregulation of these pathways. We identified nine unique macrophage, monocyte, and dendritic cell populations (Extended Data Fig. 2b, Fig. 3a). PD-L1 and IDO1 were correlated (Pearson $\left.R=0.64, p<2.2 \times 10^{-16}\right)$ and expressed to varying degrees across most of these populations and all FOVs (Fig. 3b-e, Extended Data Fig. 5a, c). Bright co-expression of both proteins was observed in CD11b+CD11 $c^{+}$macrophages, consistent with an MDSClike phenotype ${ }^{42}$ (Fig. 3d). This was also found in CD209+ DCs and CD16+ CD14+ intermediate monocytes, where it was associated with HLA-DR downregulation (Fig. 3ad). While the frequency of IDO1+ cells did not vary significantly between tissue or specimen type (Extended Data Fig. 5b), PD-L1+ cells were significantly higher in diagnostic biopsies relative to therapeutic resections, with a notable enrichment in extrapulmonary tissues (Extended Data Fig. 5b). Notably, neutrophils were also found to express IDO1 or PD-L1 (Extended Data Fig. 5d). Given that they have been shown to secrete anti-inflammatory cytokines in TB granulomas ${ }^{43}$, these findings are consistent with a regulatory effector function. Lastly, nearly $100 \%$ of multinucleated giant cells expressed IDO1 and $75 \%$ express PD-L1 (Fig. 3f). 
a

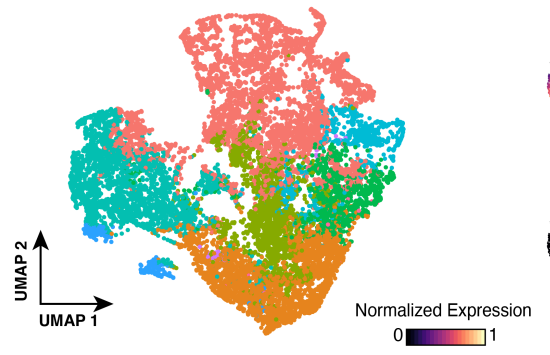

CD14+ Mono $\begin{array}{ll}\mathrm{CD} 14^{+} 16^{+} \text {Mono } & \mathrm{CD} 163^{+} \mathrm{Mac} \\ \mathrm{CD} 209^{+} \mathrm{DC}\end{array}$ CD11 $1 \mathrm{c}^{+} \mathrm{DC} / \mathrm{Mono}$ CD206 $\mathrm{Cac}^{+\mathrm{Mac}}$ Giant Cell
Granuloma myeloid cell expression profiles
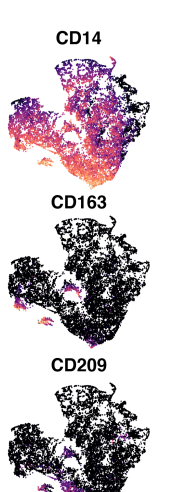

b

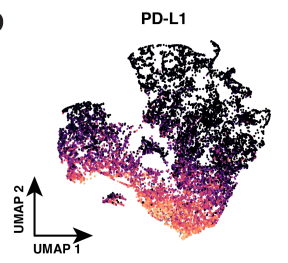

C

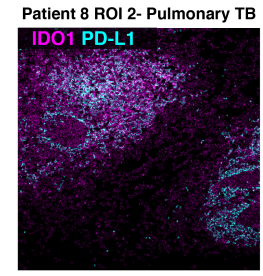

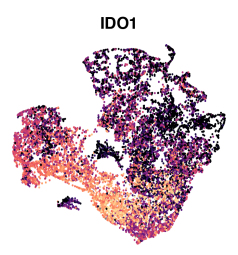

Patient 9 ROI 2- Pleural TB

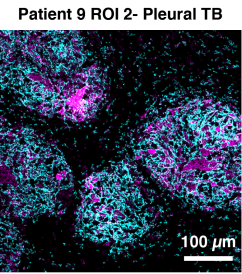

a

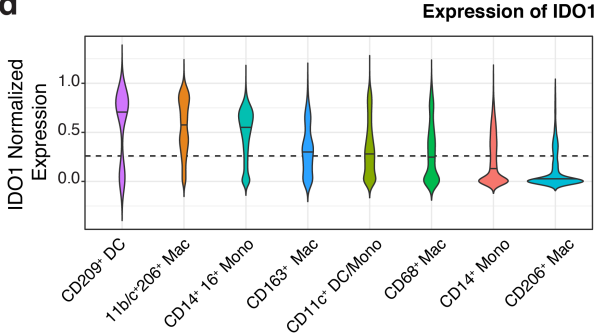

f

Expression of IDO1 and PD-L1 by giant cells Patient 8 - Pulmonary TB
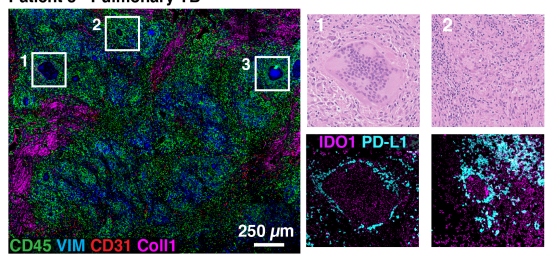

h Microenvironment expression of IDO1 and PD-L1
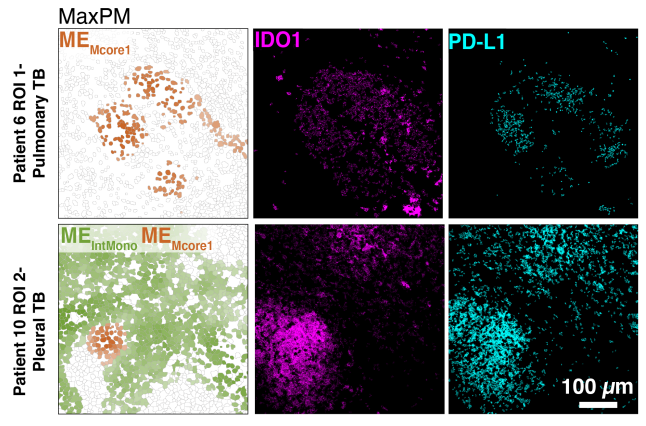
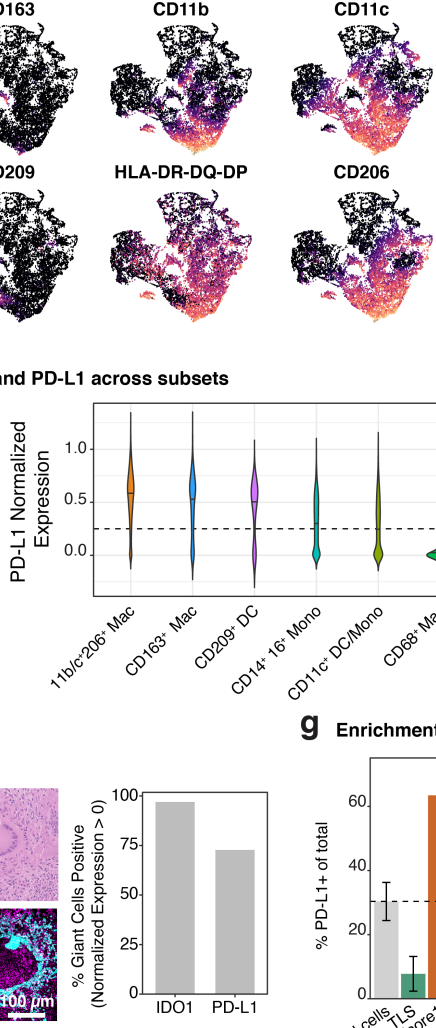

e Correlation between IDO1 and PD-L1

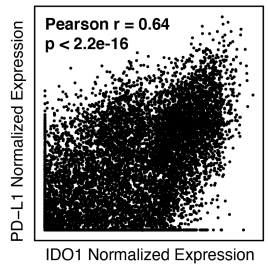

g Enrichment of PD-L1 and IDO1 expression across microenvironments
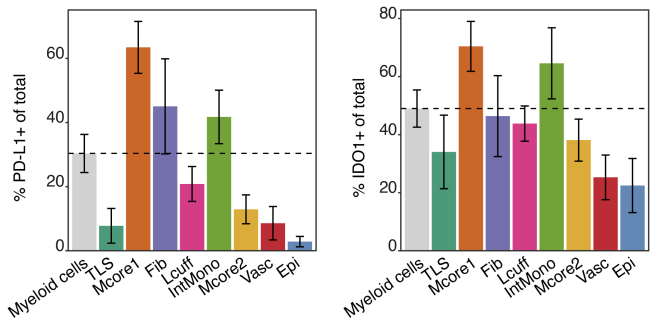

i

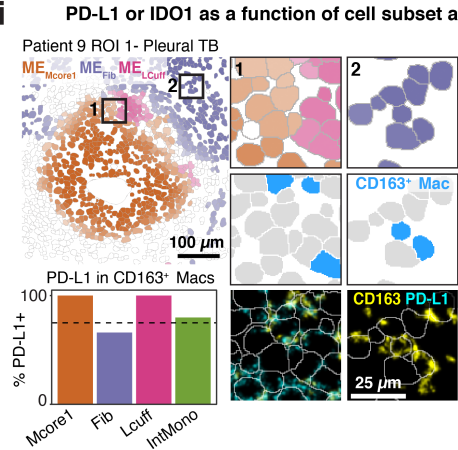

Patient 7 ROI 1- Pulmonary TB

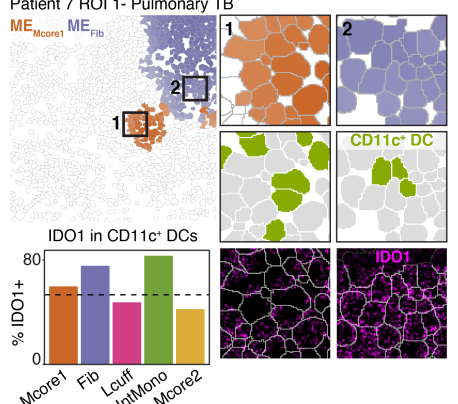

Figure 3. TB granuloma myeloid cells are characterized by spatially coordinated expression of IDO1 and PDL1. (a) UMAP visualization of all myeloid populations across all TB FOVs colored by subset (left) and normalized expression of phenotypic markers used to delineate subsets. (b) IDO1 and PD-L1 normalized expression overlaid on the UMAP. (c) Representative images of a pulmonary (left) and pleural (right) sample showing expression of IDO1 (magenta) and PD-L1 (cyan). (d) Normalized expression of IDO1 (top) and PD-L1 (bottom) for major myeloid subsets ordered by decreasing median expression value. Dashed line indicates the cutoff for positivity for IDO1 (cutoff $=0.26$ ) and PD-L1 (cutoff $=0.25$ ). (e) PD-L1 and IDO1 expression values across all myeloid cells as a biaxial scatter plot. Plot displays Pearson's $r$ and $p$-value determined by t-test. (f) Giant cells identified from a MIBI-scanned pulmonary TB sample $(C D 45=$ green, Vimentin = blue, CD31 $=$ red). Representative giant cells displayed in zoomed insets (lower left) with hematoxylin and eosin staining or IDO1 (magenta) and PD-L1 (cyan) expression. Bar plot displays the percentage of $\mathrm{IDO}^{+}$and PD-L1+ giant cells $(n=33$, normalized expression $>0)$. (g) ME Mcore1 and ME $E_{\text {IntMono }}$ max 
probability maps and representative images of a pulmonary (top) and pleural (bottom) TB sample showing expression of IDO1 (magenta) and PD-L1 (cyan). (h) The frequency of IDO1+ and PD-L1+ myeloid cells for all myeloid cells in aggregate and broken down by microenvironment. (i) The frequency of PD-L1+CD163+ macrophages (left) across MEs with a representative MaxPM. Insets are colored by ME (top), cell type (blue, middle), and CD163 (yellow) and PD-L1 (cyan) with the segmentation boundaries overlaid (white). The frequency of IDO1+CD11b+CD11 c+ macrophages (right) across MEs with a representative MaxPM. Insets are colored by ME (top), cell type (green, middle), and IDO1 (magenta) with the segmentation boundaries overlaid (white).

To determine how PD-L1 and IDO1 expression is associated with a cell's location in the granuloma, we calculated the frequency of PD-L1 ${ }^{+}$and IDO1+ cells for each cell subset in each ME (Fig. 3i, Extended Data Fig. 5e). We found that the majority of cells displayed preferential, ME-specific expression patterns. For example, the frequency of PD-L1 expressing CD163+ macrophages was highest in the ME Mcore1 $_{\text {(100\%) and MELcuff }}$ (100\%), while IDO1 expressing CD11b+ CD11c ${ }^{+}$macrophages were most enriched in $\mathrm{ME}_{\mathrm{Fib}}(75.6 \%)$ and MEIntMono (83.3\%) (Fig. 3i). Altogether, PD-L1 and IDO1 expression defines a newly identified, spatially-coordinated immunoregulatory feature of TB granulomas that supports the possibility of highly localized, myeloid-mediated immune suppression in the granuloma.

We next wanted to assess if the spatial coordination observed in tolerogenic myeloid cells extended to tolerogenic lymphocytes, like regulatory T cells (Tregs), which comprised $1.3 \%$ of all lymphocytes (Fig. 4a). Tregs (CD3 ${ }^{+} \mathrm{CD}^{+} \mathrm{Foxp}^{+}$) were preferentially enriched in MEMcore1 relative to MELcuff (Fig. 4b, $p=0.0012$ ), which stood in contrast to all other lymphocyte subsets, including Foxp3-CD4+ T cells. Furthermore, the frequency of proliferating Tregs exceeded that of all other major lymphocyte subsets (Fig. $4 c, p<0.001)$. These results suggest that Tregs and MDSC-like cells preferentially colocalize within $\mathrm{ME}_{\mathrm{Mcore}}$ to potentiate an immunomodulatory niche that could ultimately deter bacterial clearance (Fig. 4d) ${ }^{44-50}$.

Anti-inflammatory pathways like those found in MEMcore1 are often induced as a 286 form of negative feedback that moderates the cytotoxic effects of unchecked immune 287 activation ${ }^{51}$. In line with this, high expression of PD-L1 and IDO1 by granuloma myeloid 
cells would be expected to be accompanied by evidence of T cell activation in the form of checkpoint marker expression (e.g. PD-1, Lag3) ${ }^{52}$. For example, in previous work examining infiltrated triple negative breast cancer (TNBC) tumors, we found the median ratio of PD-1+: PD-L1+ immune cells to be near unity (Fig. 4f) and the prevalence of Lag3

292 or PD-1 positive lymphocytes to be $13.9 \%$ and $5.5 \%$ on average, respectively (Fig. 4 e). 293 In contrast, PD-L1+ granuloma myeloid cells far outnumbered PD-1+ lymphocytes $294\left(\log 2\left[P D-1^{+}:\right.\right.$PD-L1+] $=-5.73 \pm 3.4$, Fig. 4f). Furthermore, the small numbers of PD-1+ lymphocytes in our dataset were almost entirely restricted to METLS, consistent with $\mathrm{T}$ 296 follicular helper cells rather than an activated or exhausted phenotype (Extended Data 297 Fig. 5f). These findings are consistent with reports from the cynomolgus macaque model 298 that also found low levels of PD-1, Lag3, and CTLA4 ${ }^{14}$, suggesting that PD-L1 expression by myeloid granuloma cells occurs independently of local cytokine release by activated T cells.

a

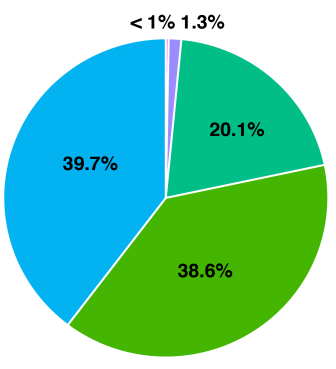

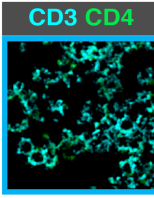

CD4 T cell CD8 T cell
B cell
Treg үo T cell
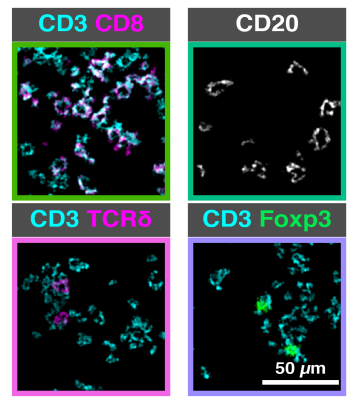

b
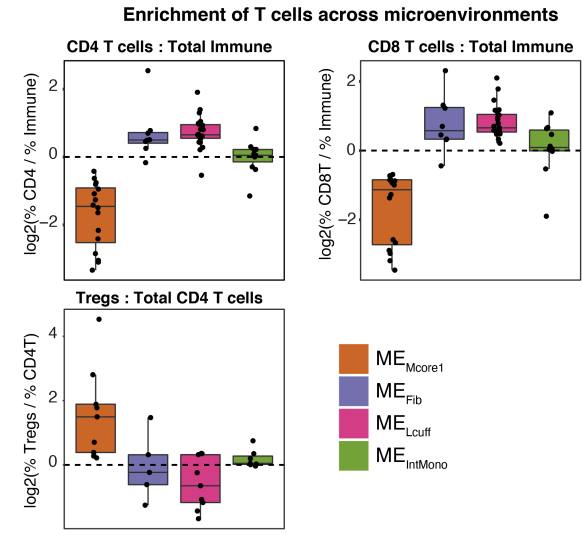

C

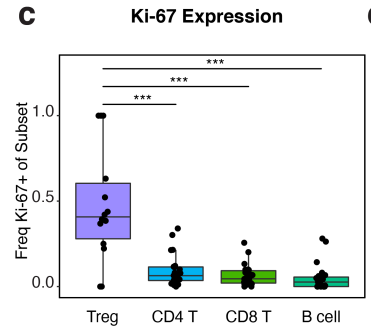

d

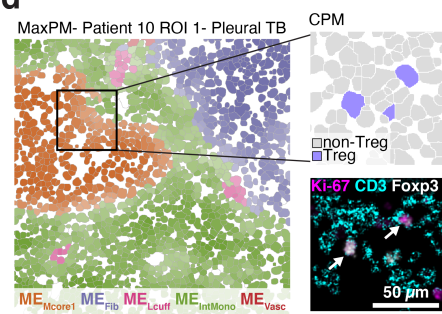

e

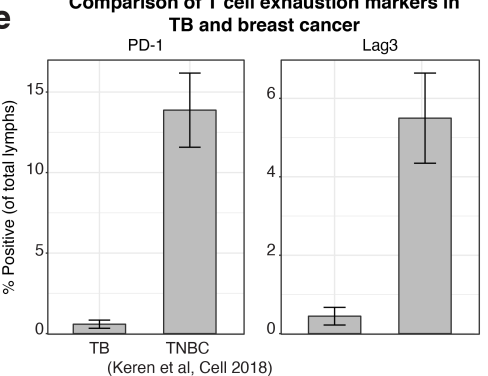

f

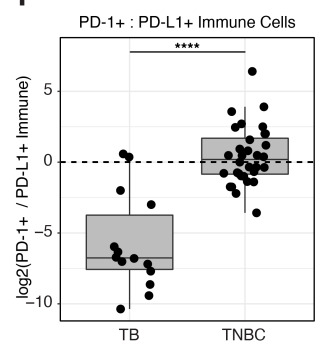

Figure 4. Granuloma lymphocytes are enriched in distinct cellular microenvironments and display a paradoxical absence of exhaustion markers. (a) Frequency of lymphocyte subsets in all TB FOVs pooled together (left) and representative images of each subset (right). (b) The frequency of CD4+ and CD8+ $\mathrm{T}$ cells relative to the frequency of total immune cells in four MEs of interest (top). The frequency of Tregs relative to the frequency of total CD4+ T cells (lower, left). (c) Frequency of Ki-67+ cells broken down by lymphocyte subset. (d) Representative image 
from a pleural TB FOV, colored by ME assignment (left). Zoomed inset displays Treg assignment (upper-right, purple = Treg, grey = non-Treg) and expression of Ki-67 (magenta), CD3 (cyan), and Foxp3 (white) (lower-right). (e) Percent of lymphocytes positive for PD-1 (left) and Lag3 (right) in all TB FOVs and TNBC. Bar represents the mean and standard error. (f) The ratio of PD-1+: PD-L1+ immune cells represented as a log2 fold-change in all TB FOVs and TNBC. All boxplots represent the median and interquartile range. All $p$-values determined with a Wilcoxon Rank Sum Test where: ns $p>0.05,{ }^{*} p<0.05,{ }^{* *} p<0.01,{ }^{* *} p<0.001,{ }^{* * *} p<0.0001$.

TB and sarcoidosis granulomas have both common and diverging features of immune regulation

In addition to being the histological hallmark of TB, granulomatous inflammation can occur in response to a foreign body or in autoimmune disorders, such as sarcoidosis ${ }^{53}$. Interestingly, several gene expression studies that have attempted to develop blood-based biomarkers for latent and active infection have struggled to differentiate between TB and sarcoidosis ${ }^{54,55}$. In order to determine to what extent the features identified here overlap with other granulomatous diseases, we compared the TB sample cohort to ten sarcoidosis cases (Extended Date Fig. 6a-b). TB lesions were more variable in composition ( $p=0.037$, Extended Date Fig. $6 \mathrm{~d}$ ) and had significantly higher frequencies of $\mathrm{CD}^{+} \mathrm{T}$ cells, neutrophils, intermediate monocytes, and giant cells (Fig. 5a, Extended Data Fig. 6b-c). Sarcoid granulomas were heavily CD4+ T cell-skewed, even relative to the CD4-skewed TB granulomas in our dataset, consistent with reports of the pathology being driven primarily by Th17 and Th1 T cells (Fig. 5b) ${ }^{56-58}$. of PD-L1+ myeloid cells (Fig. 5d, Extended Data Fig. 6e). However, unlike TB, IDO1 expression in sarcoid samples was almost entirely absent (Fig. 5d). Since we used a conservative threshold for IDO1 and PD-L1 positivity, our analysis biased toward the moderate to bright-expressing cells present in TB granulomas and control tissues. Therefore, to more comprehensively evaluate the disease specificity of PD-L1 and IDO1, we performed immunohistochemistry (IHC) for both proteins on a tissue microarray of granulomas from sarcoidosis $(n=9)$, foreign body uptake $(n=4)$, endometriosis $(n=4)$, and xanthomatosis $(n=3)$ (Extended Data Fig. 6f). We identified weak expression of IDO1 in several sarcoidosis lesions along with bright expression of PD-L1 as observed by MIBI-TOF (Extended Data Fig. 6f). However, IDO1+ and PD-L1+ cells were nearly absent in all xanthomas and endometrial lesions and rare in foreign body granulomas. 
Notably, we observed similarly high levels of IDO1 and PD-L1 in a pulmonary

a Cell types signficantly increased in TB vs sarcoidosis

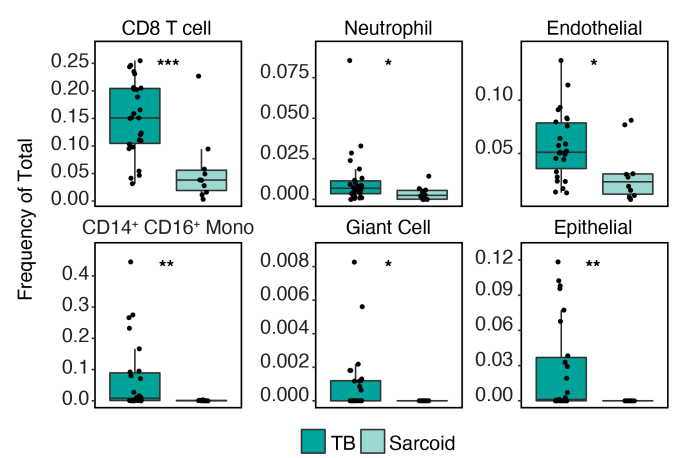

b CD4 T cell : CD8 T cell ratio TB vs sarcoidosis

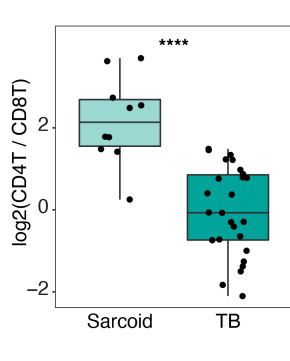

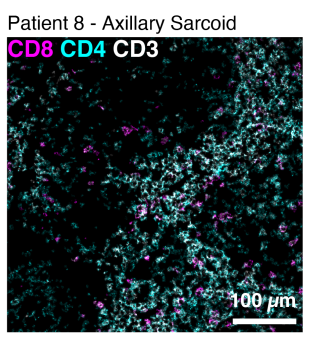

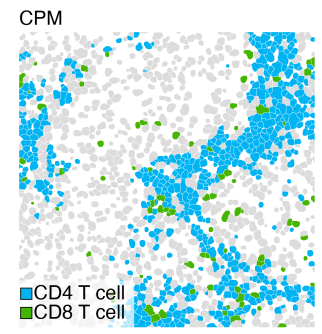

C T cell exhaustion in sarcoidosis, TB, and TNBC

d ID01 and PD-L1 Expression in TB and sarcoidosis
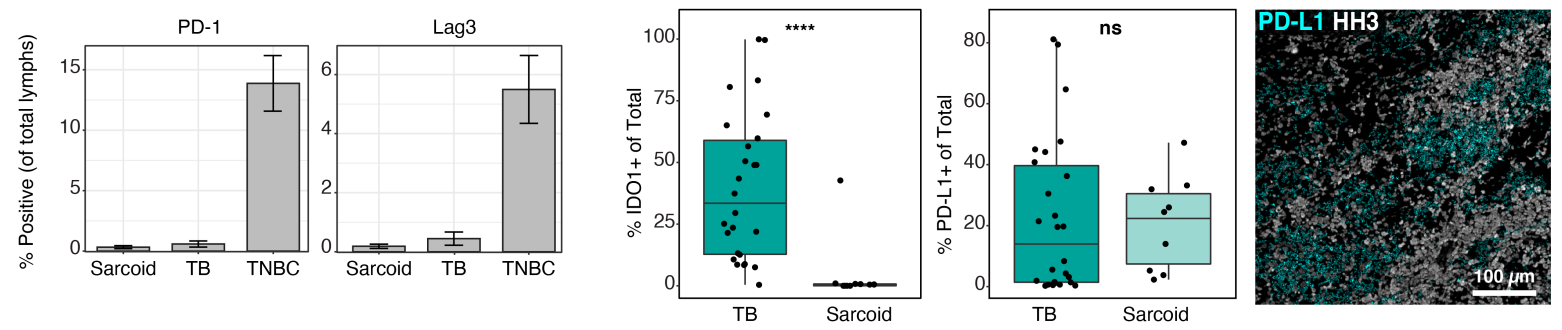

Figure 5. TB and sarcoidosis granulomas have both common and diverging features of immune regulation. (a) Frequency of cell subsets (of total cells) in TB versus sarcoidosis that are significantly different. (b) Comparison of the CD4+ $\mathrm{T}$ : CD8+ $\mathrm{T}$ cell ratio in TB versus sarcoidosis. Representative image of an axillary sarcoidosis FOV showing expression of CD8 (magenta), CD4 (cyan), and CD3 (white) (left) and colored by cell type (right, blue $=$ CD4+ T cell, green $=\mathrm{CD}^{+} \mathrm{T}$ cell) (c) Percent of lymphocytes positive for PD-1 (left) and Lag3 (right) in all sarcoidosis FOVs, TB FOVs and TNBC. Bar represents the mean and standard error. (d) Percent of total cells positive for IDO1 or PD-L1 in TB and sarcoidosis. Representative image of a sarcoidosis FOV showing expression of PD-L1 (cyan) and HH3 (white). All boxplots represent the median and interquartile range. All p-values determined with a Wilcoxon Rank Sum Test where: $n s p>0.05,{ }^{*} p<0.05,{ }^{\star \star} p<0.01,{ }^{* \star *} p<0.001,{ }^{\star \star \star *} p<0.0001$. patients where they correlate to clinical progression and treatment status MIBI-TOF study has important implications for the treatment of TB infection. However, the invasive nature of procuring solid tissue limited the cohort size and scope by biasing towards advanced infections. Moreover, the single time point per patient in our tissue 
dataset precludes temporal analysis that would correlate disease severity with granuloma immunosuppressive features. Given the clinical feasibility of venous phlebotomy and large number of publicly available blood transcriptomic datasets, we sought to correlate these features in blood from TB patients. Therefore, we used Metalntegrator to perform

a Whole blood gene expression meta-analysis

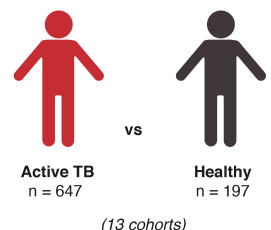

(13 cohorts) b

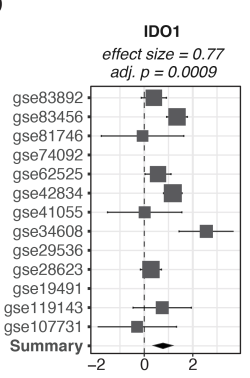

d Summary Effect Sizes Across Infection Stage
Active TB v Healthy CD274 (PD-11) PDCD1 (PD-1) effect size $=1.28$ effect size $=-0.41$ adj. $p=0.006$ Standardized Mean Difference (log2 scale)

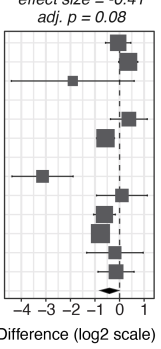

LAG3 effect size $=-0.39$

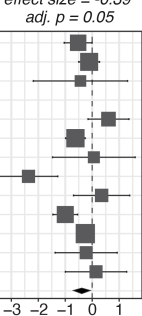

C Whole blood gene expression meta-analysis

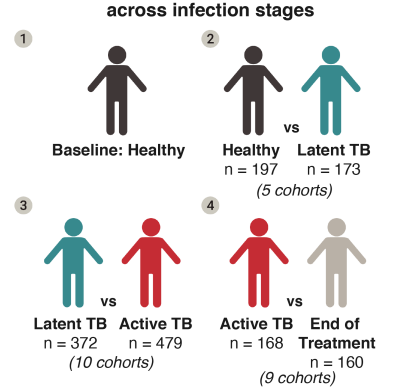

$f$

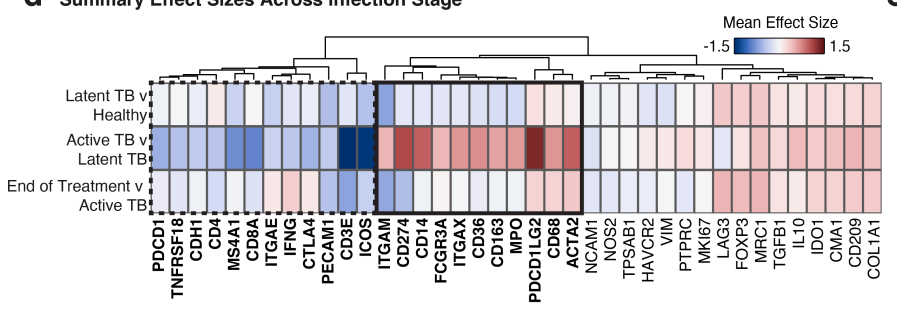

e Catalysis Treatment Response Cohort (CTRC) $f$

CD274 (PD-L1)
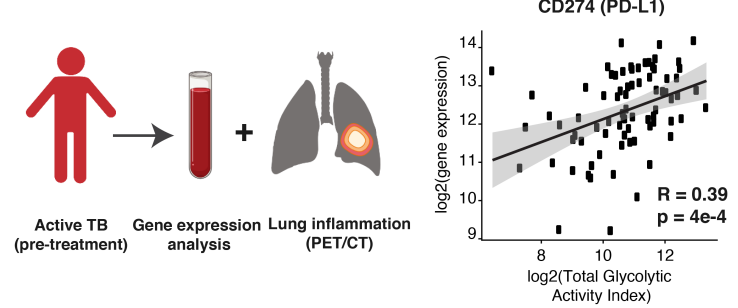

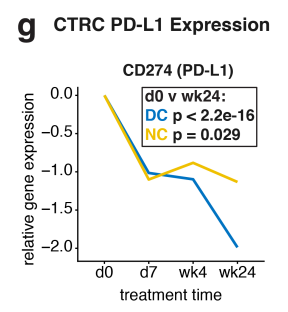

— Definite Cure — Not Cured
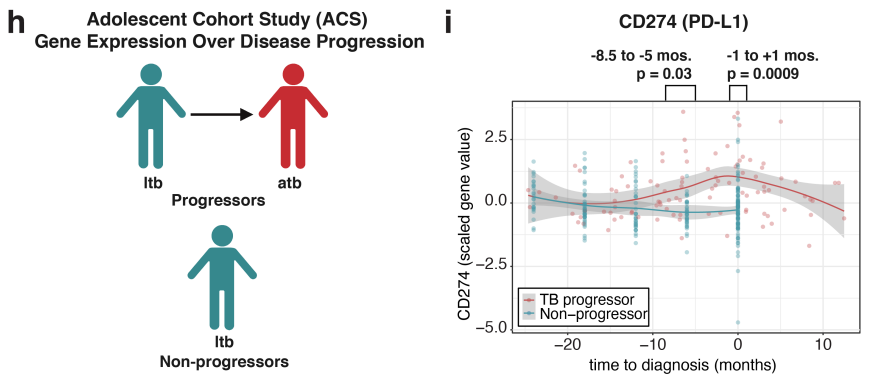

Figure 6. Immunoregulatory features of granulomas are reflected in the peripheral blood of TB patients where they correlate to clinical progression and treatment status (a) Conceptual overview of the meta-analysis of patients with active TB $(n=647)$ versus healthy controls $(n=197)$. (b) Forest plots of gene expression differences in active TB versus healthy individuals. Cohort identifiers are shown on the $y$-axis. Boxes represent the standardized mean difference in gene expression (effect size). The size of the box is proportional to the sample size of that cohort. Whiskers represent the $95 \%$ confidence interval and diamonds (black) represent the overall difference in gene expression between two groups by integrating the standardized mean differences across all cohorts. The width of the diamond corresponds to its $95 \%$ confidence interval. The adj. $p$ values ( $q$ values, FDR $5 \%$ ) for the summary effect sizes are shown above each plot. (c) Conceptual overview of gene expression analysis across clinical infection stage. (d) Heatmap of summary gene expression (mean effect size) values in latent TB $(n=173)$ versus healthy controls $(n$ $=197)$, latent TB $(n=372)$ versus active TB $(n=479)$, and active TB $(n=168)$ versus end-of-treatment $(n=160)$. Clinical stage is displayed on rows and genes are displayed across columns hierarchically clustered (Euclidean distance, complete linkage). Genes upregulated in active TB versus latent TB are shown in the solid black box, while 
downregulated genes are in the dashed black box. (e) Conceptual overview of the Catalysis Treatment Response Cohort (CTRC). (f) Correlation between PD-L1 gene expression and total glycolytic activity index (TGAI) represented as log2-transformed values. Linear fit (blue) with 95\% confidence interval (grey) displayed. A Pearson correlation of 0.39 ( $p=4 \times 10^{-4}$, t-test) is displayed below the linear fit. (g) PD-L1 gene expression across treatment time broken down by cure status (blue = definite cure and yellow = no cure). Line represents mean expression in each time point, connected across time points. P-value determined with Student's T-test for PD-L1 expression at d0 versus wk24 in the definite cure (DC, $n=71$ ) and not-cured (NC, $n=7)$ groups. (h) Conceptual overview of the Adolescent Cohort Study (ACS). (i) PD-L1 gene expression in the ACS cohort across time prior to and after diagnosis of active TB stratified by progressors (red, $n=34$ ) and non-progressors (blue, $n=109$ ). Grey silhouette represents the $95 \%$ confidence interval. P-values determined by Welch two sample t-test.

We first determined if immune regulatory features identified in granulomas could be detected in blood by comparing publicly available gene expression data of patients with active TB $(n=647)$ to healthy controls $(n=197)$ from 13 independent cohorts (Fig. 6a). In line with PD-L1 and IDO1 expression in tissue data, significant and consistent upregulation of IDO1 and CD274 (PD-L1) was found in patients with active TB infection (effect size $=0.77$ and 1.28, adj. $p=0.0009$ and 0.006 , respectively) (Fig. 6b). Additionally, checkpoint depletion in lymphoid cells was corroborated as well, with reduced expression of $P D C D 1$ (PD-1) and LAG3 observed in the blood of active TB patients (effect size $=-0.41$ and -0.39 , adj. $p=0.09$ and 0.05 respectively).

Next, we analyzed transcriptomic data from 1,549 patients across 24 cohorts in order to determine if these features were specific to active infections. In line with the solid tissue analysis, differential expression of genes associated with regulatory myeloid cells (e.g. PDL1, PDL2, CD11b, CD11c, CD163) or T-cell immune checkpoint (e.g. PD1, CTLA4) delineated active from latent infections (Fig. 6d). Moreover, the majority of these genes returned to baseline levels seen in healthy controls after completing antimicrobial therapy (Fig. 6d, Extended Data Fig. 6h). Taken together, these results suggest a shift toward myeloid-mediated immune suppression that is specifically manifested during active TB infection.

Because PD-L1 gene expression exhibited the largest effect size relative to healthy controls, we next analyzed the Catalysis Treatment Response Cohort (CTRC) to determine if PD-L1 could be used as a biomarker for estimating disease burden and predicting clearance of infection (Fig 6e). Patients enrolled in this study provided venous blood and underwent PET/CT imaging61,62. Expression of PD-L1 at diagnosis was found to be directly correlated with total glycolytic activity index (TGAI), a radiographic metric 
373 for quantifying lung inflammation (Fig. 6f, Pearson $r=0.39 p=4 \times 10^{-4}$ ). 24 weeks after

374 treatment, patients were clinically classified into four groups: definite cure, probable cure,

375 possible cure, and not cured. Relative to the time of diagnosis, the reduction in PD-L1

376 expression in definitely cured patients $(n=71)$ were 2 times greater on average than in

377 uncured patients ( $\mathrm{n}=7$, Fig. $6 \mathrm{~g}$ ). A nearly identical trend was observed for PD-L2 as well

378 (PDCDLG2, Extended Data Fig. 6i).

379 Lastly, we analyzed the Adolescent Cohort Study (ACS) to determine if PD-L1 could 380 be used for predicting progression to active disease. Latently infected individuals enrolled 381 in this study underwent regular blood collection and were clinically monitored for 382 symptoms of active infection (Fig. 6h) ${ }^{63,64}$. PD-L1 transcript levels were significantly 383 elevated in progressors $(n=34)$ relative to non-progressors $(n=109) 8.5$ months prior to 384 progression $(p=0.03$ ) with the peak occurring at time of diagnosis $(p=0.0009)$ (Fig. 6i). 385 Taken together, these results raise the intriguing possibility for using PD-L1 expression 386 in peripheral blood as a predictive biomarker for early intervention in latently infected 387 individuals.

\section{Discussion}

After nearly 140 years of research into the pathophysiology of human TB infection, central questions remain unresolved, in large part because granuloma formation and 392 progression are very difficult to emulate in tractable animal models. One of the most 393 critical questions that remains unanswered is which immune mechanisms drive 394 progression from latent infection to symptomatic active TB disease, the source of 11 395 million new cases and 1.5 million fatalities each year ${ }^{1}$. Furthermore, individual granuloma fate can vary significantly between lesions within individuals, raising questions about the 397 local immune dynamics that influence a granuloma's capacity to control infection while 398 mitigating tissue damage. With this in mind we used MIBI-TOF to identify recurrent 399 features of immune regulation in archival tissue from patients with active TB infection. 400 This spatial cell atlas allowed us to relate granuloma structure and composition. We 401 identified 19 unique cell subsets that preferentially organize into eight reoccurring cellular 402 microenvironments. TB granulomas appear to follow a consistent structural outline 
403 characterized by spatially coordinated expression of PD-L1 and IDO1, myeloid core404 infiltrating Tregs, and a striking absence of T cell activation as measured through PD-1 405 and Lag3. Some of these features, such as high expression of PD-L1 and presence of 406 MDSC-like macrophages, were present in non-infectious granulomas as well pointing to 407 certain universal immune programs associated with the granulomatous immune 408 response. However, even compared to sarcoidosis, foreign body uptake, xanthomatosis, 409 and endometrial lesions, spatially coordinated expression of IDO1 and PD-L1 was unique 410 to mycobacterial granulomas.

411 Previous studies in the cynomolgus macaque model of TB have demonstrated that 412 granulomas from a single individual can have disparate outcomes with respect to bacterial 413 burden and inflammatory trajectory ${ }^{10,11}$. The variation we see in our imaging dataset 414 suggests these local outcomes may be driven in part by unique cellular infiltrate and 415 structure within each granuloma. We observed that certain features, such as a high 416 frequency of $\mathrm{CD}^{+} \mathrm{T}$ cells, corresponded with reduced levels of more differentiated 417 macrophage phenotypes, a profile consistently present in therapeutic resections where 418 PD-L1 expression was also diminished. Since CD8 ${ }^{+} \mathrm{T}$ cells have been shown to be 419 critical for clearance of TB infection ${ }^{65,66}$, understanding the immunological environments 420 that promote CD8 ${ }^{+} \mathrm{T}$ cell activity could reveal novel insights into immune features critical 421 for bacterial clearance.

$422 \quad$ By leveraging 29 publicly-available gene expression studies of over 1500 TB 423 patients and healthy controls, we were able to identify these immunoregulatory signatures 424 in peripheral blood and correlate them with disease burden and clinical outcome. Genes 425 found in solid tissue to be overexpressed at the protein level by immunosuppressive 426 myeloid cells (PD-L1, IDO1, CD163) were upregulated in blood. Similarly, genes 427 associated with T-cell activation were downregulated, consistent with the rare incidence 428 of PD-1 or Lag3 expression in tissue. Importantly, the magnitude of these trends was 429 distinctly higher in patients with active disease relative to those with latent or treated 430 infections. The highest effect size of genes measured in our analysis was observed for 431 PD-L1. PD-L1 was found to correlate with pulmonary disease burden and appears to be 432 a prognostic biomarker of progression from latent to active TB disease. Taken together, 
433 the work presented here reveals new aspects of immune regulation in TB infection that

434 have important implications for understanding disease pathogenesis and improving 435 clinical management.

The high levels of PD-L1 and IDO1 observed in the near absence of PD-1 offers clues into how the immunosuppressive niche during human infection is initiated and maintained. These findings are consistent with a TGF $\beta$ or IL-10 driven process where production of these cytokines can suppress inflammation, promote immunosuppression, and induce peripheral regulatory $\mathrm{T}$ cell differentiation and proliferation ${ }^{67-70}$. This is supported by recent work in mice where focal secretion of TGF- $\beta$ within the myeloid core was suggested to preferentially suppress neighboring T cells and in non-human primates where granuloma formation was associated with IL-10 secretion ${ }^{71,72}$.

Both IDO1 and PD-L1 have been shown to dampen anti-tumor immune responses

in cancer, which has prompted the development of host-directed immunotherapies ${ }^{73}$. Our

446 findings suggest that similar approaches could be used to block PD-L1 mediated immune suppression to promote bacterial clearance. However, evidence of T-cell activation or exhaustion is not present in our dataset or in the cynomolgus macaque model. This suggests that unlike checkpoint blockade in the setting of cancer, the efficacy of PD-L1 or PD-1 blockade could differ significantly. Recent reports of TB reactivation following PD-

4511 blockade illustrate the seemingly paradoxical effects that can occur with host directed 452 therapies and emphasize the need to comprehensively map the temporal and spatial 453 dynamics of these pathways ${ }^{74-76}$. In line with this, a critical next step will be to extend this 454 work to relate these features to bacterial burden, inflammatory dynamics, and granuloma 455 age in a primate model that accurately recapitulates human TB pathology.

To the best of our knowledge, this is the most comprehensive systems analysis of

457 TB to date. We identified dynamics of cellular composition and immunoregulatory 458 pathways in TB granulomas that are reflected in the peripheral immune response to TB. 459 These results have implications both for developing host-directed immunotherapies and 460 for identifying patients at risk of progression to active disease or treatment failure. 461 Expression of proteins such as IDO1 and PD-L1 aligns with immune evasion mechanisms 462 observed in tumor-immune microenvironment. The interface of granuloma and tumor 
463 immunobiology offers vast opportunities to explore how tactics of immune evasion in 464 tumors may contribute to bacterial persistence in granulomas. Future multiplexed 465 imaging studies of granulomas from controlled TB exposures will offer novel insights on 466 how these local regulatory dynamics influence granuloma fate, and ultimately, infection 467 outcome.

\section{Data and Code Availability}

470 All custom code used to analyze data can be accessed at the following link: 471 https://github.com/efrancis28/TBMIBI. All processed images and annotated single cell 472 data will be made available on Mendeley Data.

\section{Methods}

\section{Human Samples}

477 Human samples were acquired in accordance with IRB protocol \# 46646.

\section{Tuberculosis Granuloma Cohort}

480 Formalin-fixed Paraffin-embedded (FFPE) Mtb infected tissues were acquired from 481 Stanford Health Care's tissue repository from seven patients undergoing a pre-treatment 482 diagnostic biopsy. Tissues were screened to include those that were positive for Acid483 Fast Bacillus (AFB+) and Mtb DNA by polymerase chain reaction (PCR). Archival surgical 484 resection tissues were acquired from University of KwaZulu-Natal, Inkosi Albert Luthuli 485 Central Hospital from six patients with Mtb infection who underwent therapeutic resection 486 of infected tissue due to infection severity or treatment failure. This specimen group 487 contained $\mathrm{HIV}^{+}$patients on antiretroviral therapy $(n=3)$, HIV- patients $(n=2)$, and one 488 patient with no HIV infection reported. All clinical details for specimens can be found in 489 Extended Data Table 1. $5 \mu \mathrm{m}$ serial sections of each specimen were stained with 490 hematoxylin and eosin (H\&E) and inspected by an anatomic pathologist to screen for the 491 presence of granulomatous inflammation. Regions with histologically solid granulomas or 492 cellular granulomatous inflammation were included. Regions with excessive fibrosis or 
493 necrosis were excluded. Two $500 \mu \mathrm{m}^{2}$ fields of view (FOV) were chosen from each tissue 494 block for imaging.

\section{Non-tuberculous Granulomas and Controls Tissues}

497 Regions of granulomatous inflammation from FFPE sarcoidosis and foreign body 498 reactions from Stanford Health Care were chosen by an anatomic pathologist. $0.5 \mathrm{~mm}$ 499 cores were selected and constructed into a tissue microarray (TMA). A pulmonary 500 Mycobacterium avium case was acquired from Stanford Health Care through selection 501 criteria of positive Acid-Fast Bacillus (AFB+) staining and PCR positivity for M. avium 502 Complex (MAC). A $5 \mu \mathrm{m}$ serial section of this specimen was stained with hematoxylin and 503 eosin (H\&E) and inspected by an anatomic pathologist to screen for the presence of 504 granulomatous inflammation. Control tissues of FFPE tonsil, spleen, and placenta were 505 acquired from Stanford Health Care. Small regions of each tissue were selected and 506 placed in a TMA.

\section{Antibody Preparation}

509 Antibodies were conjugated to isotopic metal reporters as described previously ${ }^{19}$. 510 Following conjugation antibodies were diluted in Candor PBS Antibody Stabilization 511 solution (Candor Bioscience). Antibodies were either stored at $4^{\circ} \mathrm{C}$ or lyophilized in 100 $512 \mathrm{mM} \mathrm{D}-(+)$-Trehalose dehydrate (Sigma Aldrich) with ultrapure distilled $\mathrm{H}_{2} \mathrm{O}$ for storage at $513-20^{\circ} \mathrm{C}$. Prior to staining, lyophilized antibodies were reconstituted in a buffer of Tris 514 (Thermo Fisher Scientific), sodium azide (Sigma Aldrich), ultrapure water (Thermo Fisher 515 Scientific), and antibody stabilizer (Candor Bioscienc) to a concentration of $0.05 \mathrm{mg} / \mathrm{mL}$. 516 Information on the antibodies, metal reporters, and staining concentrations is located in 517 Extended Data Table 1.

\section{Tissue Staining}

520 Tissues were sectioned (5 $\mu \mathrm{m}$ section thickness) from tissue blocks on gold and tantalum521 sputtered microscope slides. Slides were baked at $70{ }^{\circ} \mathrm{C}$ overnight followed by 522 deparaffinization and rehydration with washes in xylene (3x), 100\% ethanol (2x), 95\% 
523 ethanol (2x), 80\% ethanol ( $1 \mathrm{x}), 70 \%$ ethanol ( $1 \mathrm{x})$, and $\mathrm{ddH}_{2} \mathrm{O}$ with a Leica ST4020 Linear

524 Stainer (Leica Biosystems). Tissues next underwent antigen retrieval by submerging 525 sides in 3-in-1 Target Retrieval Solution ( $\mathrm{pH} 9$, DAKO Agilent) and incubating at $97^{\circ} \mathrm{C}$ for

52640 minutes in a Lab Vision PT Module (Thermo Fisher Scientific). After cooling to room 527 temperature slides were washed in 1X PBS IHC Washer Buffer with Tween 20 (Cell 528 Marque) with $0.1 \%(\mathrm{w} / \mathrm{v})$ bovine serum albumin (Thermo Fisher). Next, all tissues 529 underwent two rounds of blocking, the first to block endogenous biotin and avidin with an 530 Avidin/Biotin Blocking Kit (Biolegend). Tissues were then washed with wash buffer and 531 blocked for 1 hour at room temperature with 1x TBS IHC Wash Buffer with Tween 20 with $5323 \%(\mathrm{v} / \mathrm{v})$ normal donkey serum (Sigma-Aldrich), $0.1 \%(\mathrm{v} / \mathrm{v})$ cold fish skin gelatin (Sigma 533 Aldrich), $0.1 \%(\mathrm{v} / \mathrm{v})$ Triton X-100, and 0.05\% (v/v) Sodium Azide. The first antibody 534 cocktail was prepared in 1x TBS IHC Wash Buffer with Tween 20 with 3\% (v/v) normal 535 donkey serum (Sigma-Aldrich) and filtered through a $0.1 \mu \mathrm{m}$ centrifugal filter (Millipore) 536 prior to incubation with tissue overnight at $4{ }^{\circ} \mathrm{C}$ in a humidity chamber. Following the 537 overnight incubation slides were washed twice for 5 minutes in wash buffer. The second 538 day antibody cocktail was prepared as described and incubated with the tissues for 1 539 hour at $4^{\circ} \mathrm{C}$ in a humidity chamber. Following staining, slides were washed twice for 5 540 minutes in wash buffer and fixed in a solution of $2 \%$ glutaraldehyde (Electron Microscopy 541 Sciences) solution in low-barium PBS for 5 minutes. Slides were washed in PBS (1x), 0.1

$542 \mathrm{M}$ Tris at $\mathrm{pH} 8.5(3 \mathrm{x}), \mathrm{ddH}_{2} \mathrm{O}(2 \mathrm{x})$, and then dehydrated by washing in $70 \%$ ethanol $(1 \mathrm{x})$, $54380 \%$ ethanol (1x), 95\% ethanol (2x), and $100 \%$ ethanol $(2 x)$. Slides were dried under 544 vacuum prior to imaging.

\section{MIBI-TOF Imaging}

547 Imaging was performed using a MIBI-TOF instrument with a Hyperion ion source. $\mathrm{Xe}^{+}$

548 primary ions were used to sequentially sputter pixels for a given FOV. The following

549 imaging parameters were used:

550

551 - Acquisition setting: $80 \mathrm{kHz}$ 
- Field size: $500 \mu \mathrm{m}^{2}$ (TB, M. avium and controls) or $450 \mu \mathrm{m}^{2}$ (sarcoidosis) at 1024 x 1024 pixels

- Dwell time: $4 \mathrm{~ms}$

- Median gun current on tissue: $1.45 \mathrm{nA} \mathrm{Xe^{+ }}$

- Ion dose: $3.38 \mathrm{nAmp}$ hours / $\mathrm{mm}^{2}$ for $500 \mu \mathrm{m}^{2}$ FOVs and $3.75 \mathrm{nAmp}$ hours / mm² for $450 \mu \mathrm{m}^{2}$

558

\section{Low-level Image Processing}

560 Multiplexed image sets were extracted, slide background-subtracted, denoised, and 561 aggregate filtered as previously described ${ }^{19}$. All parameters for these steps can be found 562 in Extended Data Table 1. In addition to these processing steps, image compensation was performed to account for signal spillover due to adducts and oxides for the following 564 interferences: Collagen-1 to IDO1 and Lag3, H3K9Ac to panCK and MPO, Chym/Tryp to MPO, Ki67 to CD209, CD20 to CD16, CD16 to IFN $\gamma$, CD11c to IDO1, and HLA-DR-DQDP to CD11b.

\section{Single Cell Segmentation}

569 Nuclear segmentation was performed using an adapted version of the DeepCell ${ }^{19-21}$ CNN 570 architecture. Training data was generated from MIBI-TOF images of human melanoma 571 that were stained with $\mathrm{HH} 3$ to identify nuclei and $\mathrm{Na}^{+} \mathrm{K}+\mathrm{ATPase}$ to identify the cell 572 membrane. Color overlays of these two channels were used to manually segment nuclei 573 in ImageJ. This generated training data with labels for the nuclear interior, nuclear border, 574 and non-nuclear background. Training data was generated for 5 images to train the 575 network architecture. Images were divided into overlapping crops of 64x64 pixels. Each 576 crop was randomly flipped, rotated, and sheared during training to further augment the 577 available data. Stochastic gradient descent was used to train the network, combined with 578 early stopping to prevent over-fitting. Following training all MIBI-TOF images from our 579 cohorts were provided as input to the network to predict the class of each pixel: nuclear 580 interior, nuclear border, or non-nuclear background. The nuclear interior probability map 581 for each image was thresholded and segmented as described previously ${ }^{19}$ followed by a 
582 3-pixel radial expansion around each nucleus to define the cell object boundaries. A correction was applied to FOVs that contained multinucleated giant cells (MGNs). First each MGN was identified using a combination of HH3, CD45, and Vimentin and manually segmented in ImageJ to produce a binary mask of each MGN. All pixels within the binary mask were reassigned to belong to the MGN cell object(s).

\section{Single Cell Phenotyping and Composition}

589 Single cell data was extracted for all cell objects and area-normalized. Cells with a sum of less than 0.1 area-normalized counts across all lineage channels were excluded from analysis. Single cell data was linearly scaled with a scaling factor of 100 and asinhtransformed with a co-factor of 5 . All mass channels were scaled to $99.9^{\text {th }}$ percentile. In order to assign each cell to a lineage, the FlowSOM clustering algorithm was used in iterative rounds with the Bioconductor "FlowSOM" package in $\mathrm{R}^{22}$. The first clustering round separated cells into four major lineages using the "Metaclustering_consensus" function: immune, epithelial, fibroblast, and endothelial. Immune cells were then clustered again to delineate $\mathrm{B}$ cells, CD4+ $\mathrm{T}$ cells, $\mathrm{CD} 8^{+} \mathrm{T}$ cells, Tregs, neutrophils, mast cells, and mononuclear phagocytes (macrophages, monocytes, and dendritic cells). Immune cells with an expression profile not consistent with any of those subsets were annotated as 'other immune.' Lastly, the mononuclear phagocytes were clustered to 25 metaclusters which were merged into 7 groups. Giant cells were manually identified. $\gamma \delta \mathrm{T}$ cells were annotated as T cells with CD3 signal greater than or equal to the mean expression of CD4 ${ }^{+}$T cells and TCR $-\delta$ signal $>0.5$ normalized expression. CD163 macrophages were identified as those with CD163 signal > 0.5 normalized expression. The relative abundance of all major lineages was determined out of total cells per FOV and the relative frequency of immune cell subsets was determined out of total immune cells per FOV.

\section{Immune Cell Composition Clustering and Cell Type Association Analysis}

609 The Pearson correlation coefficient was calculated between all pairs of TB FOVs based 610 on the relative frequency of all immune cell subsets. The coefficients were used to 611 hierarchically cluster the FOVs using complete linkage and a distance metric of 1- 
612 correlation coefficient. To identify consensus clusters the percent variance explained was

613 measured across a range of 1-10 clusters. The elbow point of this plot was used to

614 determine the optimal number of clusters. A randomized dataset was produced to

615 compare to the observed clustering by randomizing the frequency values across immune

616 cell subsets within each FOV. This dataset was also clustered using the Pearson

617 correlation coefficient and compared with the observed result. To assess the significance

618 of co-occurrence of cell types, a chi-square test was run between all cell type pairs using

619 the counts of each cell type across all TB FOVs. The resulting p-values were adjusted

620 using a false discovery rate (FDR) of $5 \%$.

621

\section{Protein Enrichment Analysis}

623 A spatial enrichment approached was used as previously described ${ }^{19}$ to identify patterns

624 of protein enrichment or exclusion across all protein pairs. $\mathrm{HH} 3, \mathrm{Na}^{+} \mathrm{K}^{+} \mathrm{ATP}$ ase, and HLA

625 Class 1 were excluded from the analysis. For each pair of markers, $\mathrm{X}$ and $\mathrm{Y}$, the number

626 of times cells positive for protein $X$ was within a $\sim 50$ um radius of cells positive for protein

$627 Y$ was counted. Thresholds for positivity were customized to each marker individually

628 using a silhouette scanning approach from the MetaCyto software in $R^{77}$. A null

629 distribution was produced by performing 1000 bootstrap permutations where the locations

630 of cells positive for protein $Y$ were randomized. A z-score was calculated comparing the

631 number of true cooccurrences of cells positive for protein $X$ and $Y$ relative to the null

632 distribution. For each pair of proteins $X$ and $Y$ the average $z$-score was calculated across

633 all TB FOVs. Next, all positive enrichments between protein pairs (average Z score $>0$, 634 excluded self-self protein enrichment scores) were used to produce a weighted, 635 undirected network where the nodes are the individual markers and the edge weights are 636 proportional to the average z-score (higher z-score $\rightarrow$ shorter edge length). The leading 637 eigenvector algorithm for community detection was used to identify protein enrichment 638 communities in this network ${ }^{78}$.

639

640 Spatial Latent Dirichlet Allocation 
641 Spatial-LDA is an adaptation of Latent Dirichlet Allocation (LDA), a machine learning 642 approach used to model topics in text documents, where topics consist of words with a 643 high probability of cooccurrence, allowing mapping of topics to abstract definitions (ex. 644 ['dog', 'frog', 'horse] $\rightarrow$ 'animals'). Spatial-LDA builds on this paradigm by representing 645 CPMs as documents and cell types as words. Spatial-LDA was conducted to identify 646 topics (here referred to as microenvironments) across all TB FOVs. Cell types with fewer 647 than 100 cells across the entire cohort were excluded from analysis $(\gamma \delta \mathrm{T}$ cells and 648 CD209+DC). Furthermore, multinucleated giant cells were excluded due to their cell size. 649 Spatial-LDA was implemented as described ${ }^{30}$ with $d=1000$, a spatial radius $r=50 \mu \mathrm{m}$ 650 to complement the protein enrichment analysis, and a microenvironment (ME) number of $651 \mathrm{n}=8$. The ME number was determined empirically. For each FOV a maximum probability 652 map (MaxPM) was produced by classifying each cell to the microenvironment with the 653 highest probability and coloring that cell by its microenvironment and probability. The cell 654 type preferences for each ME were determined by assessing the mean probability for all 655 cell types across all MEs. The mean expression for each functional marker across MEs 656 was determined by weighting protein expression by ME probability and calculating the 657 mean of weighted expression values across markers and MEs. The mean probability for 658 all MEs was determined for all FOVs (average of single cell values) and used to conduct 659 a Principal Component Analysis (PCA). The clustering approach described for immune 660 cell frequency clusters (above) was applied to ME frequencies across FOVs to identify 661 the optimal number of ME clusters to capture the maximal variance in our dataset.

\section{Identification of the Myeloid Core}

664 In order to assess which microenvironments represented the histologically defined 665 myeloid core, binary masks of the myeloid core were generated for 18/26 FOVs. The 666 masks were generated by first combining the signal of CD11c, CD11b, CD14, CD206, 667 CD68, and PD-L1. The combined images were imported into ImageJ and hand-annotated 668 using ROI annotation tools. The annotated ROI was exported as a binary mask. This 669 mask was further processed in Matlab to close any holes, exclude objects smaller than 6701000 pixels, and dilate the mask to smooth edges. Next cells were assigned to belonging 
671 to the myeloid core if they had complete overlap with the binary mask. Cells on the mask

672 boundary or outside of the mask were designated as 'non-myeloid core.' The proportion

673 of cells in the myeloid core was assessed across each ME for the 18 FOVs and a MEs

674 with a median frequency in the myeloid core $>50 \%$ were designated as myeloid core

675 MEs.

676

677

Myeloid Cell UMAP Visualization

678 UMAP embeddings were determined for all non-granulocytic myeloid cells using the $R$

679 implementation ${ }^{79}$ with the following parameters: $n \_$neighbors $=15$, min_dist $=0.1$ and 680 the following markers: CD45, CD68, CD206, CD11c, CD11b, CD14, CD16, CD209, and 681 CD163.

\section{Immunoregulatory Protein Analysis}

684 Positivity thresholds for IDO1, PD-L1, PD-1, and Lag3 were automatically determined as described above. Immune control tissues tonsil, spleen, and placenta were used to validate antibody performance. Correlation between IDO1 and PD-L1 was determined across the entire cohort and subsets of specimens using Pearson correlation analysis. The frequency of cells positive for IDO1 and PD-L1 were enumerated across all subsets. To assess PD-L1 and IDO1 positivity with respect to ME and cell subset, the total number of cells across all myeloid subsets per ME was pooled across all FOVs. The quantity of cells for each subset positive for IDO1 or PD-L1 was determined per ME. Any ME with < $1 \%$ of the total for a subset was excluded from analysis. PD-1 and Lag3 expression were analyzed on lymphocytes or total immune cells. PD-1 and Lag3 were also analyzed on immune cells from a human Triple Negative Breast Cancer (TNBC) cohort that was previously published by our group ${ }^{19}$. Positivity for PD-1 and Lag3 for TNBC immune cells was determined as described in the originally published study.

\section{Cell Composition Analysis of Sarcoidosis and Tuberculosis}

699 Single cells from sarcoidosis FOVs were segmented as described above. Single cell data 
701 were included in the described FlowSOM clustering procedure. To compare the cellular

702 diversity of TB with sarcoidosis the Shannon Diversity Index was calculated using the 703 counts of all cell subsets per TB or sarcoidosis FOV.

Immunohistochemistry of PD-L1 and IDO1

706 Immunohistochemistry (IHC) for PD-L1 and IDO1 was performed using the antibody 707 reagents in Extended Data Table 1 at a concentration of $1 \mu \mathrm{g} / \mathrm{mL}$. The IHC protocol 708 mirrors the MIBI-TOF protocol with the addition of blocking endogenous peroxidase 709 activity with $3 \% \mathrm{H}_{2} \mathrm{O}_{2}$ (Sigma Aldrich) in $\mathrm{ddH}_{2} \mathrm{O}$ after epitope retrieval. On the second day 710 of staining, instead of proceeding with the MIBI-TOF protocol, tissues were washed twice 711 for 5 minutes in wash buffer and stained using the ImmPRESS universal (Anti712 Mouse/Anti-Rabbit) kit (Vector labs).

\section{Whole Blood Transcriptomic Analysis}

715 Publicly available gene expression data sets (Extended Data Table 2) were collected, 716 annotated, and used for meta-analysis conducted using Metalntegrator ${ }^{59}$. Gene 717 expression matrices were prepared for each dataset to determine effect sizes for genes 718 of all proteins included in the MIBI-TOF analysis and an additional set of genes with 719 similar biological function, such as ICOS and CTLA4. Summary effect sizes were 720 calculated to assess gene expression differences across clinical groups (healthy, active 721 TB, latent TB, end of treatment, TB progression, and during treatment). For the Catalysis 722 Treatment Response Cohort (CTRC) gene expression measurements at diagnosis of TB 723 were correlated with matched Total Glycolytic Activity Index (TGAI), a readout of PET-CT

724 activity. A linear regression was fit between CD274 gene expression and TGAl and the 725 correlation was assessed with Pearson correlation analysis. To assess CD274 and 726 PDCDLG2 gene expression over treatment, expression values were normalized to the 727 measurement taken at diagnosis (d0). Gene expression data in the Adolescent Cohort 728 Study (ACS) were separated by progression status. Local regression (LOESS) was used 729 to fit the gene expression data over time in each group. The significance of separation 
730 between progressors and non-progressors was determined in two different time intervals

731 using a Student's t-test.

732

\section{Software}

734 Image processing was conducted with Matlab 2016a and Matlab 2019b. Statistical 735 analysis was conducted in Matlab 2016a, Matlab 2019b, and R. Data visualization and 736 plots were generated in R. Representative images were processed in Adobe Photoshop 737 and figures were prepared in Adobe Illustrator. Schematic visualizations were produced 738 with Biorender.

\section{Author Contributions:}

741 E.F.M conceived the study design, performed experiments, analyzed data, and wrote the 742 manuscript. M.D. conducted the blood transcriptomics analysis. L.K. assisted with 743 analysis conceptualization. Z.C. and V.J. implemented the spatial-LDA analysis. L.K, 744 A.D., N.F.G., A.B., and W.G. assisted with data analysis. M.B. assisted with assay 745 development. D.V.V. developed Deepcell. M.F., P.K.R., E.F., M.V.D.R., N.B., and 746 A.J.C.S. provided samples and consulted on tissue cohort design. S.C.B, P.K., and M.A. 747 supervised the work.

\section{Acknowledgments}

750 The authors thank Tyler Risom, David Glass, Matthew Carter, and Anne Kasmar for 751 discussions and comments. The authors thank Pauline Chu and the Stanford Human 752 Histology Core for providing technical assistance. E.F.M was supported by the NSF 753 Graduate Research Fellowship (grant no. 2017242837). L.K. was a Damon Runyon 754 Fellow supported by the Damon Runyon Cancer Research Foundation (DRG-2292-17) 755 and a non-stipendiary awardee of the EMBO Long-Term fellowship (ALTF 1128-2016). 756 Noah Greenwald was supported by F31CA246880. A.J.C.S. was supported 757 R61/33Al138280, R01Al134810, the CRDF Global, the South African Medical Research 758 Council, and an NRF BRICS Multilateral grant to A.J.C.S. M.A. was supported by 1-DP5759 OD019822. S.C.B. and M.A. were jointly supported by 1R01AG056287 and 
1R01AG057915, 1U24CA224309, the Bill and Melinda Gates Foundation, and a

761 Translational Research Award from the Stanford Cancer Institute. S.J.G. was supported by U19 Al104209, R01 AR067145, and R01 Al32494.

\section{Conflicts of Interest}

M.A. and S.C.B. are inventors on patent US20150287578A1. M.A. and S.C.B. are board members and shareholders in IonPath Inc. E.F.M. has previously consulted for lonPath

767 Inc.

\section{References}

1. WHO I Global tuberculosis report 2019. WHO (2020).

2. Cohen, S. B. et al. Alveolar Macrophages Provide an Early Mycobacterium tuberculosis Niche and Initiate Dissemination. Cell Host Microbe 24, 439-446.e4 (2018).

3. Wolf, A. J. et al. Mycobacterium tuberculosis Infects Dendritic Cells with High Frequency and Impairs Their Function In Vivo . J. Immunol. 179, 2509-2519 (2007).

4. Bold, T. D. \& Ernst, J. D. Who benefits from granulomas, mycobacteria or host? Cell 136, 17-9 (2009).

5. Davis, J. M. \& Ramakrishnan, L. The Role of the Granuloma in Expansion and Dissemination of Early Tuberculous Infection. Cell 136, 37-49 (2009).

6. Ramakrishnan, L. Revisiting the role of the granuloma in tuberculosis. Nat. Rev. Immunol. 12, (2012).

7. Cadena, A. M., Fortune, S. M. \& Flynn, J. L. Heterogeneity in tuberculosis. (2017) doi:10.1038/nri.2017.69.

8. Subbian, S. et al. Lesion-Specific Immune Response in Granulomas of Patients with Pulmonary Tuberculosis: A Pilot Study. PLoS One 10, e0132249 (2015).

9. Coleman, M. T. et al. Early Changes by (18)Fluorodeoxyglucose positron emission tomography coregistered with computed tomography predict outcome after Mycobacterium tuberculosis infection in cynomolgus macaques. Infect. Immun. 82, 2400-4 (2014).

10. Lin, P. L. et al. Sterilization of granulomas is common in active and latent tuberculosis despite within-host variability in bacterial killing. Nat. Med. 20, 75-79 (2013).

11. Martin, C. J. et al. Digitally Barcoding Mycobacterium tuberculosis Reveals In Vivo Infection Dynamics in the Macaque Model of Tuberculosis. MBio 8, e00312-17 (2017).

12. Carow, B. et al. Spatial and temporal localization of immune transcripts defines hallmarks and diversity in the tuberculosis granuloma. Nat. Commun. 10, 1-15 (2019). 
13. Marakalala, M. J. et al. Inflammatory signaling in human tuberculosis granulomas is spatially organized. Nat. Med. 22, 531-538 (2016).

14. Wong, E. A. et al. Low levels of T cell exhaustion in tuberculous lung granulomas. Infect. Immun. 86, (2018).

15. Kauffman, K. D. et al. Defective positioning in granulomas but not lung-homing limits CD4 T-cell interactions with Mycobacterium tuberculosis-infected macrophages in rhesus macaques. Mucosal Immunol. 11, 462-473 (2018).

16. Keren, L. et al. MIBI-TOF: A multiplexed imaging platform relates cellular phenotypes and tissue structure. Sci. Adv. 5, eaax5851 (2019).

17. Krishnan, N., Robertson, B. D. \& Thwaites, G. The mechanisms and consequences of the extra-pulmonary dissemination of Mycobacterium tuberculosis. Tuberculosis vol. 90 361-366 (2010).

18. Ranaivomanana, P. et al. Cytokine Biomarkers Associated with Human ExtraPulmonary Tuberculosis Clinical Strains and Symptoms. Front. Microbiol. 9, 275 (2018).

19. Keren, L. et al. A Structured Tumor-Immune Microenvironment in Triple Negative Breast Cancer Revealed by Multiplexed Ion Beam Imaging. Cell 174, 13731387.e19 (2018).

20. Van Valen, D. A. et al. Deep Learning Automates the Quantitative Analysis of Individual Cells in Live-Cell Imaging Experiments. PLOS Comput. Biol. 12, e1005177 (2016).

21. Bannon, D. et al. Dynamic allocation of computational resources for deep learning-enabled cellular image analysis with Kubernetes. bioRxiv 505032 (2019) doi:10.1101/505032.

22. Van Gassen, S. et al. FlowSOM: Using self-organizing maps for visualization and interpretation of cytometry data. Cytom. Part A 87, 636-645 (2015).

23. Flynn, J. L., Chan, J. \& Lin, P. L. Macrophages and control of granulomatous inflammation in tuberculosis. Mucosal Immunology vol. 4 271-278 (2011).

24. Garcia-Rodriguez, K. M., Goenka, A., Alonso-Rasgado, M. T., Hernández-Pando, R. \& Bulfone-Paus, S. The role of mast cells in tuberculosis: Orchestrating innate immune crosstalk? Frontiers in Immunology vol. 8 (2017).

25. Ratnam, S., Ratnam, S., Puri, B. K. \& Chandrasekhar, S. Mast cell response during the early phase of tuberculosis: an electron microscopic study. Can. J. Microbiol. 23, 1245-1251 (1977).

26. Carlos, D. et al. Mast Cells Modulate Pulmonary Acute Inflammation and Host Defense in a Murine Model of Tuberculosis. J. Infect. Dis. 196, 1361-1368 (2007).

27. Polena, H. et al. Mycobacterium tuberculosis exploits the formation of new blood vessels for its dissemination. Sci. Rep. 6, 1-11 (2016).

28. Oehlers, S. H. et al. Interception of host angiogenic signalling limits mycobacterial growth. Nature 517, 612-615 (2015).

29. Girvan, M. \& Newman, M. E. J. Community structure in social and biological networks. Proc. Natl. Acad. Sci. U. S. A. 99, 7821-7826 (2002).

30. Chen, Z., Soifer, I., Hilton, H., Keren, L. \& Jojic, V. Modeling Multiplexed Images with Spatial-LDA Reveals Novel Tissue Microenvironments . J. Comput. Biol. 
(2020) doi:10.1089/cmb.2019.0340.

31. Shi, J. et al. PD-1 Controls Follicular T Helper Cell Positioning and Function. Immunity 49, 264-274.e4 (2018).

32. Dieu-Nosjean, M. C., Goc, J., Giraldo, N. A., Sautès-Fridman, C. \& Fridman, W. $\mathrm{H}$. Tertiary lymphoid structures in cancer and beyond. Trends in Immunology vol. 35 571-580 (2014).

33. Ulrichs, T. et al. Human tuberculous granulomas induce peripheral lymphoid follicle-like structures to orchestrate local host defence in the lung. J. Pathol. 204, 217-228 (2004).

34. Difazio, R. M. et al. Active transforming growth factor- $\beta$ is associated with phenotypic changes in granulomas after drug treatment in pulmonary tuberculosis. DARU, J. Pharm. Sci. 24, 6 (2016).

35. Krystel-Whittemore, M., Dileepan, K. N. \& Wood, J. G. Mast cell: A multifunctional master cell. Frontiers in Immunology vol. 6 (2016).

36. Shen, L. et al. PD-1/PD-L pathway inhibits M.tb-specific CD4+ T-cell functions and phagocytosis of macrophages in active tuberculosis. Sci. Rep. 6, 38362 (2016).

37. Shen, L. et al. The characteristic profiles of PD-1 and PD-L1 expressions and dynamic changes during treatment in active tuberculosis. Tuberculosis 101, 146150 (2016).

38. Mehra, S. et al. Granuloma correlates of protection against tuberculosis and mechanisms of immune modulation by Mycobacterium tuberculosis. J. Infect. Dis. 207, 1115-27 (2013).

39. Munn, D. H. \& Mellor, A. L. IDO in the Tumor Microenvironment: Inflammation, Counter-Regulation, and Tolerance. Trends in Immunology vol. 37 193-207 (2016).

40. Gautam, U. S. et al. In vivo inhibition of tryptophan catabolism reorganizes the tuberculoma and augments immune-mediated control of Mycobacterium tuberculosis. Proc. Natl. Acad. Sci. U. S. A. 115, E62-E71 (2018).

41. Jurado, J. O. et al. Programmed death (PD)-1:PD-ligand 1/PD-ligand 2 pathway inhibits T cell effector functions during human tuberculosis. J. Immunol. 181, 11625 (2008).

42. Human myeloid derived suppressor cell (MDSC) subset phenotypes I The Journal of Immunology. https://www.jimmunol.org/content/198/1_Supplement/211.2.

43. Gideon, H. P., Phuah, J., Junecko, B. A. \& Mattila, J. T. Neutrophils express proand anti-inflammatory cytokines in granulomas from Mycobacterium tuberculosisinfected cynomolgus macaques. Mucosal Immunol. 12, 1370-1381 (2019).

44. Kanamori, M., Nakatsukasa, H., Okada, M., Lu, Q. \& Yoshimura, A. Induced Regulatory T Cells: Their Development, Stability, and Applications. Trends in Immunology vol. 37 803-811 (2016).

45. Hsu, P. et al. IL-10 Potentiates Differentiation of Human Induced Regulatory $T$ Cells via STAT3 and Foxo1. J. Immunol. 195, 3665-3674 (2015).

46. Llopiz, D. et al. IL-10 expression defines an immunosuppressive dendritic cell population induced by antitumor therapeutic vaccination. Oncotarget 8, 2659- 
2671 (2017).

47. Ribeiro-Rodrigues, R. et al. A role for CD4+CD25+ T cells in regulation of the immune response during human tuberculosis. Clin. Exp. Immunol. 144, 25-34 (2006).

48. Scott-Browne, J. P. et al. Expansion and function of Foxp3-expressing T regulatory cells during tuberculosis. J. Exp. Med. 204, 2159-2169 (2007).

49. Guyot-Revol, V., Innes, J. A., Hackforth, S., Hinks, T. \& Lalvani, A. Regulatory T cells are expanded in blood and disease sites in patients with tuberculosis. Am. J. Respir. Crit. Care Med. 173, 803-810 (2006).

50. Green, A. M. et al. CD4 + Regulatory T Cells in a Cynomolgus Macaque Model of Mycobacterium tuberculosis Infection . J. Infect. Dis. 202, 533-541 (2010).

51. Bagaitkar, J. Cellular dynamics of resolving inflammation. Blood vol. 124 17011703 (2014).

52. Wherry, E. J. \& Kurachi, M. Molecular and cellular insights into T cell exhaustion. Nature Reviews Immunology vol. 15 486-499 (2015).

53. Baughman, R. P., Lower, E. E. \& Du Bois, R. M. Sarcoidosis. in Lancet vol. 361 1111-1118 (Elsevier Limited, 2003).

54. Koth, L. L. et al. Sarcoidosis blood transcriptome reflects lung inflammation and overlaps with tuberculosis. Am. J. Respir. Crit. Care Med. 184, 1153-1163 (2011).

55. Maertzdorf, J. et al. Common patterns and disease-related signatures in tuberculosis and sarcoidosis. Proc. Natl. Acad. Sci. U. S. A. 109, 7853-7858 (2012).

56. Wahlström, J. et al. Analysis of intracellular cytokines in CD4+ and CD8+ lung and blood T cells in sarcoidosis. Am. J. Respir. Crit. Care Med. 163, 115-121 (2001).

57. Rossi, G. A. et al. Helper T-lymphocytes in pulmonary sarcoidosis. Functional analysis of a lung T-cell subpopulation in patients with active disease. Am. Rev. Respir. Dis. 133, 1086-1090 (1986).

58. Facco, M. et al. Sarcoidosis is a Th1/Th17 multisystem disorder. Thorax 66, 144150 (2011).

59. Haynes, W. A. et al. Empowering multi-cohort gene expression analysis to increase reproducibility. in Pacific Symposium on Biocomputing vol. 0 144-153 (World Scientific Publishing Co. Pte Ltd, 2017).

60. Sweeney, T. E., Haynes, W. A., Vallania, F., loannidis, J. P. \& Khatri, P. Methods to increase reproducibility in differential gene expression via meta-analysis. Nucleic Acids Res. (2017) doi:10.1093/nar/gkw797.

61. Roy Chowdhury, R. et al. A multi-cohort study of the immune factors associated with M. tuberculosis infection outcomes. Nature vol. 560 644-648 (2018).

62. Malherbe, S. T. et al. Persisting positron emission tomography lesion activity and Mycobacterium tuberculosis mRNA after tuberculosis cure. Nat. Med. 22, 10941100 (2016).

63. Scriba, T. J. et al. Sequential inflammatory processes define human progression from M. tuberculosis infection to tuberculosis disease. PLOS Pathog. 13, e1006687 (2017). 
933

64. Zak, D. E. et al. A blood RNA signature for tuberculosis disease risk: a prospective cohort study. Lancet 387, 2312-2322 (2016).

65. Flynn, J. L., Goldstein, M. M., Triebold, K. J., Koller, B. \& Bloom, B. R. Major histocompatibility complex class I-restricted $T$ cells are required for resistance to Mycobacterium tuberculosis infection. Proc. Natl. Acad. Sci. U. S. A. 89, 1201312017 (1992).

66. Lalvani, A. et al. Human cytolytic and interferon $\mathrm{Y}$-secreting CD8+ T lymphocytes specific for Mycobacterium tuberculosis. Proc. Natl. Acad. Sci. U. S. A. 95, 270275 (1998).

67. Salazar-Onfray, F., López, M. N. \& Mendoza-Naranjo, A. Paradoxical effects of cytokines in tumor immune surveillance and tumor immune escape. Cytokine Growth Factor Rev. 18, 171-182 (2007).

68. Li, M. O., Sanjabi, S. \& Flavell, R. A. A. Transforming Growth Factor- $\beta$ Controls Development, Homeostasis, and Tolerance of T Cells by Regulatory T CellDependent and -Independent Mechanisms. Immunity 25, 455-471 (2006).

69. Jarnicki, A. G., Lysaght, J., Todryk, S. \& Mills, K. H. G. Suppression of Antitumor Immunity by IL-10 and TGF- $\beta$-Producing T Cells Infiltrating the Growing Tumor: Influence of Tumor Environment on the Induction of CD4 + and CD8 + Regulatory T Cells . J. Immunol. 177, 896-904 (2006).

70. Letterio, J. J. \& Roberts, A. B. REGULATION OF IMMUNE RESPONSES BY TGF-ß. Annu. Rev. Immunol. 16, 137-161 (1998).

71. Gern, B. H. et al. TGF $\beta$ restricts T cell function and bacterial control within the tuberculous granuloma. bioRxiv 696534 (2019) doi:10.1101/696534.

72. Wong, E. A. et al. IL-10 Impairs Local Immune Response in Lung Granulomas and Lymph Nodes during Early Mycobacterium tuberculosis Infection . J. Immunol. 204, 644-659 (2020).

73. Havel, J. J., Chowell, D. \& Chan, T. A. The evolving landscape of biomarkers for checkpoint inhibitor immunotherapy. Nature Reviews Cancer vol. 19 133-150 (2019).

74. Barber, D. L. et al. Tuberculosis following PD-1 blockade for cancer immunotherapy. Sci. Transl. Med. 11, (2019).

75. Anastasopoulou, A., Ziogas, D. C., Samarkos, M., Kirkwood, J. M. \& Gogas, H. Reactivation of tuberculosis in cancer patients following administration of immune checkpoint inhibitors: Current evidence and clinical practice recommendations. Journal for ImmunoTherapy of Cancer vol. 7239 (2019).

76. Tezera, L. B. et al. Anti-PD-1 immunotherapy leads to tuberculosis reactivation via dysregulation of TNF-a. Elife 9, (2020).

77. Hu, Z. et al. MetaCyto: A Tool for Automated Meta-analysis of Mass and Flow Cytometry Data. Cell Rep. 24, 1377-1388 (2018).

78. Newman, M. E. J. Finding community structure in networks using the eigenvectors of matrices. Phys. Rev. E - Stat. Nonlinear, Soft Matter Phys. 74, (2006).

79. Mclnnes, L., Healy, J. \& Melville, J. UMAP: Uniform Manifold Approximation and Projection for Dimension Reduction. (2018). 